Asymmetric Electrochemical Polymerization in Cholesteric Liquid Crystalline Media: Effect of Isomeric Structures of Chiral Inducers Containing Bornyl Group

Atsushi Matsumura, Fan Yang, Hiromasa Goto*

Synthetic Metals, 202, 157-164 (2015).

Asymmetric Electrochemical Polymerization in Cholesteric Liquid Crystalline Media: Effect of Isomeric Structures of Chiral Inducers Containing Bornyl Group

Atsushi Matsumura ${ }^{1}$, Yang $_{\text {Fan }}{ }^{1}$, Hiromasa Goto ${ }^{1, *}$

${ }^{1}$ Division of Materials Science, Faculty of Pure and Applied Sciences, University of Tsukuba, Tsukuba, Ibaraki 305-8573, Japan

Correspondence to H. Goto, e-mail: gotoh@ims.tsukuba.ac.jp

Highlights
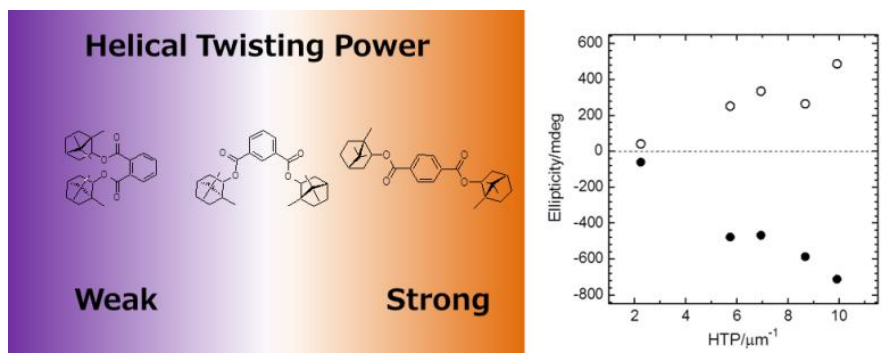

Chiral inducers were synthesized from $L$-borneol.

Helical twisting power depends on molecular geometry of the chiral inducers.

CD intensity of the PEDOT films depends on HTP of the chiral inducers.

The PEDOT film (PF-para) is electro-chiroptically active. 


\title{
Asymmetric Electrochemical Polymerization in Cholesteric Liquid Crystalline Media: Effect of Isomeric Structures of Chiral Inducers Containing Bornyl Group
}

\author{
Atsushi Matsumura ${ }^{1}$, Yang Fan $^{1}$, Hiromasa Goto ${ }^{1, *}$ \\ ${ }^{1}$ Division of Materials Science, Faculty of Pure and Applied Sciences, University of \\ Tsukuba, Tsukuba, Ibaraki 305-8573, Japan
}

Correspondence to H. Goto, e-mail: gotoh@ims.tsukuba.ac.jp

\begin{abstract}
Five chiral inducers were synthesized with $L$-borneol and benzenecarboxylic acids. These chiral inducers have the same substituent (bornyl ester) but different geometries. Para-substituted chiral inducer (I-p) shows relatively high macroscopic helical twisting power $\left(\beta_{\mathrm{M}}\right)$, while ortho-substituted chiral inducer (I-o) shows lower. This difference is related with the geometric similarity between the chiral inducers and the host liquid crystals. The result also suggested that distortion of $\pi$ system induced by polar solvents causes low $\beta_{\mathrm{M}}$ of the I-o. Optically active poly(3,4-ethylenedioxythiophene) (PEDOT) films were chemically prepared in cholesteric liquid crystal (CLC) electrolyte solution induced by the chiral inducers. Circular dichroism (CD) intensities of the resultant polymer films are almost proportional to helical twisting power (HTP) of the chiral inducers. Optical rotatory dispersion (ORD) spectra of the polymer film prepared in the CLC electrolyte induced by I-p (PF-para) shows isosbestic point during the oxidation. This indicates that electro-chiroptically active polymer film was obtained through chiral induction of the bornyl chiral inducer.
\end{abstract}

Keywords; chiral inducer, structural isomer, asymmetric electrochemical polymerization

\section{Introduction}

Highly-ordered structure in polymers has a great advantage for obtaining macroscopic properties such as ionic conduction [1], mechanical properties [2] and characteristic morphorogies [3]. Many approaches for controlling highly-ordered structure of conjugated polymers have been examined [4-7]. Highly-ordered conjugated polymers have attraction in their energy transferring and optical properties [8-10], forming mesoscopically characteristic structure such as lamellae [11], nanofibers [12] and one-handed helical aggregations [13].

Asymmetric electrochemical polymerization prepares conjugated polymer films that have one-handed helical aggregation [14]. Optically active polymer films can be prepared from achiral monomers [15,16]. In this method, cholesteric liquid crystals (CLCs), a chiral liquid crystal with one-handed helical mesoscopic structure, are employed as reaction solvents. The molecular arrangement of CLCs can produce helical polymer backbones during polymerization [17]. Circular dichroism (CD) spectra of polymer films synthesized in a CLC solvent often show bisignate Cotton effect 
Asymmetric Electrochemical Polymerization in Cholesteric Liquid Crystalline Media: Effect of Isomeric Structures of Chiral Inducers Containing Bornyl Group

Atsushi Matsumura, Fan Yang, Hiromasa Goto*

Synthetic Metals, 202, 157-164 (2015).

corresponding to a maximum optical absorption band due to chiral exciton coupling of polymer backbones via Davydov splitting. Thus, bisignate Cotton effect implies formation of chiral aggregation of polymer backbones in the $\pi$-conjugated system.

A monomer with large length/diameter ratio is advantageous to formation of helical aggregation [18] because the chiroptical property of the resultant films depends on excluded volume interaction between the monomer and CLC molecules. We are reporting studies of the relationships among the molecular geometry of monomers and the properties of polymer films.

CLCs for the asymmetric electrochemical polymerization are often prepared by addition of a chiral inducer (a chiral dopant) to a nematic liquid crystal. One-handed helical structures of CLCs are compared according to twisting ability. In asymmetric electrochemical polymerization, the chiral inducer should be carefully chosen because macroscopic properties of the polymer film depend on environment produced by CLC electrolyte $[18,19]$. Cholesterol oleyl carbonate has been used as a chiral inducer in CLC electrolytes [16-18], but pure aliphatic chiral compounds tend to have low twisting ability because of conformational flexibility of the molecules [20]. For increasing the twisting ability, designed chiral inducers such as three-rings-type [19], chiral-octyl-type [21] and double-stereogenic-center-type [22] have been studied for effective asymmetric electrochemical polymerization. Few studies have mentioned the relationship between geometry of chiral inducers and properties of polymer films.

We synthesized five chiral inducers containing bornyl group (Scheme 1). Optically active poly(3,4-ethylenedioxythiophene) (PEDOT) films were then prepared by asymmetric electrochemical polymerization in CLC electrolyte solution with these chiral inducers. The bornyl group is a carbon skeleton of borneol and isoborneol. We employ $L$-borneol to introduce the bornyl group [23,24] into the chiral inducers. Due to their stereochemistry, borneol derivatives have rigidity at substituted position [25]. Since the flexibility of alkyl groups can decrease the twisting ability, rigidity is one of the molecular design strategies for chiral inducers [26,27]. Hence, a chiral inducer having bornyl group is expected to enhance twisting ability. Bornyl group has already been used for liquid crystalline polymers [28] and chiral inducers [29]. However, the chiral inducer containing bornyl group in the previous study showed low twisting ability compared to cholesterol oleyl carbonate. To increase twisting ability, we employed benzenecarboxylic moiety for enhancement of an interaction between chiral inducers and liquid crystalline hosts. Since $L$-borneol has no aromatic group, we expect benzenecarboxylic moiety to improve the solute-solvent $\pi-\pi$ interaction, improving the twisting ability of the chiral inducer [19,30]. Moreover, benzenecarboxylic acids have simple symmetric structure, favorable for predicting conformation of the chiral inducers during the polymerization.

\section{Experimental}

\section{Instrumentation and materials}

Chemical structures of chiral inducers as shown in Scheme 1 were confirmed by ${ }^{1} \mathrm{H}$ and ${ }^{13} \mathrm{C}$ NMR spectroscopies (JEOL JNM-ECS 400). The chemical shifts in deutrated chloroform $\left(\mathrm{CDCl}_{3}\right)$ were reported against tetrametylsilane (TMS) as an internal standard. UV-vis absorption spectra were performed with a UV-3100PC (Shimadzu). $\mathrm{CD}$, optical rotatory dispersion (ORD) and linear dichroism (LD) measurements were performed with a J-720 (JASCO). LD of the polymer films was checked and removed 
Asymmetric Electrochemical Polymerization in Cholesteric Liquid Crystalline Media: Effect of Isomeric Structures of Chiral Inducers Containing Bornyl Group

Atsushi Matsumura, Fan Yang, Hiromasa Goto*

Synthetic Metals, 202, 157-164 (2015).

from the CD spectra by using artifact removal system (JASCO) [31]. CD spectra of PF-para during oxidation process were measured before UV-vis absorption measurement during oxidation process. In this optical measurement, same polymer film was used. Concentrations of the chiral inducers in $n$-hexane and acetonitrile solutions were $0.1 \mathrm{mM}$ for optical measurements. Infrared (IR) absorption spectra were performed with a FT/IR 550 (JASCO) using KBr method. Cyclic voltammetry (CV) measurements were carried out with a $\mu$ AUTOLAB TYPE Ш (ECO Chemie, the Netherlands). Acetonitrile electrolyte solution containing $0.1 \mathrm{M}$ tetrabutylammonium perchlorate (TBAP) was used for the electrochemical measurements. Twisting ability of the chiral inducers were measured with the Cano-wedge cell (EHC, KCRK-03, $\tan \theta=$ 0.0083). Optical texture observations were carried out using a Nikon ECLIPS LV 100 high resolution polarizing optical microscope (POM) with a Nikon LU Plan Fluor lens and a Nikon CFIUW lens.

All reagents were used without further purification. $L$-borneol was purchased from ACROS ORGANICS (USA). TBAP, isophthalic acid, phthalic anhydride, $N, N$-dimethyl-4-aminopyridine (DMAP) were purchased from Tokyo Kasei (TCI, Japan). $\quad N, N$-Dicyclohexylcarbodiamide (DCC) and terephthalic acid were purchased from Wako Pure Chemical (Japan). 4-Cyano-4'-n-hexylbiphenyl (6CB) was purchased from Merck (USA). Acetonitrile, $n$-hexane, dichrolomethane (DCM), ethyl acetate were purchased from Nacalai Tesque (Japan). Silica gel $60 \mathrm{~N}$ was purchased from Kanto Chemicals (Japan).

\section{Synthesis of 1,7,7-trimethylbicyclo[2.2.1]hept-2-ol benzoate (I-s)}

Benzoic acid (0.3664 g, $3.0 \mathrm{mmol})$, L-borneol (1.3882 g, $9.0 \mathrm{mmol})$, DCC (0.6190 g, $3.0 \mathrm{mmol})$, DMAP $(0.0360 \mathrm{~g}, 0.3 \mathrm{mmol})$ were mixed in $5 \mathrm{~mL}$ of DCM at room temperature and stirred for $20 \mathrm{~h}$. The mixture was extracted with DCM, and dried over magnesium sulfate, then purified by silica gel column chromatography (eluent: hexane/etyl acetate $=1 / 4)$ to afford pale yellow oil $(0.3772 \mathrm{~g}, 1.46 \mathrm{mmol}, 48.7 \%)$.

${ }^{1} \mathrm{H}$ NMR (400 MHz; $\mathrm{CDCl}_{3}$; TMS): $\delta 0.918(\mathrm{~s}, 6 \mathrm{H}), 0.971$ (s, 3H) 1.10.2-1.145 (dd, $1 \mathrm{H}$, $J=3.6,14.0 \mathrm{~Hz}), 1.281-1.442(\mathrm{~m}, 2 \mathrm{H}), 1.729-1.826(\mathrm{~m}, 2 \mathrm{H}), 2.111-2.178(\mathrm{~m}, 1 \mathrm{H})$, 2.449-2.512 (m, 1H), 5.101-5.140 (m, 2H), 7.431-7.469 (m, 2H), 7.536-7.576 (m, 1H), 8.053-8.077 (m, 2H). ${ }^{13} \mathrm{C}$ NMR (100 MHz; $\mathrm{CDCl}_{3}$; TMS): $\delta$ 13.62, 18.94, 19.74, 27.40, $28.10,36.93,45.00,47.89,49.10,80.52,128.33,129.51,130.91,132.75,166.83$.

\section{Synthesis of di(1,7,7-trimethylbicyclo[2.2.1]hept-2-ol)terephthalate (I-p)}

Quantity used: Terephthalic acid (0.2494 g, $1.5 \mathrm{mmol})$, -borneol (1.3867 g, $9.0 \mathrm{mmol})$, DCC (0.6203 g, $3.0 \mathrm{mmol})$, DMAP (0.0381 g, $0.3 \mathrm{mmol})$, DCM $(5 \mathrm{~mL}), 22 \mathrm{~h}$. Yield = $63.0 \%$ (0.4144 g, $0.94 \mathrm{mmol}) .{ }^{1} \mathrm{H}$ NMR (400 MHz; $\mathrm{CDCl}_{3}$; TMS): $\delta 0.921(\mathrm{~s}, 3 \mathrm{H})$, $0.926(\mathrm{~s}, 3 \mathrm{H}), 0.977(\mathrm{~s}, 3 \mathrm{H}), 1.112-1.156(\mathrm{dd}, 1 \mathrm{H}, J=3.2,14.0 \mathrm{~Hz}), 1.255-1.349(\mathrm{~m}$, $2 \mathrm{H}), 1.394-1.456(\mathrm{~m}, 1 \mathrm{H}), 1.745-1.837(\mathrm{~m}, 2 \mathrm{H}), 2.086-2.154(\mathrm{~m}, 1 \mathrm{H}), 2.465-2.525(\mathrm{~m}$, 1H), 5.119-5.158 (m, 1H), $8.128(\mathrm{~s}, 2 \mathrm{H}) .{ }^{13} \mathrm{C} \mathrm{NMR}$ (100 MHz; $\mathrm{CDCl}_{3}$; TMS): $\delta 13.62$, $18.92,19.73,27.39,28.10,36.89,44.97,47.93,49.15,81.09,129.46,134.50,166.07$.

\section{Synthesis of di(1,7,7-trimethylbicyclo[2.2.1]hept-2-ol)isophthalate (I-m)}

Quantity used: Isophthalic acid $(0.2490 \mathrm{~g}, 1.5 \mathrm{mmol}), L$-borneol $(1.3842 \mathrm{~g}, 9.0 \mathrm{mmol})$, DCC (0.6245 g, $3.0 \mathrm{mmol})$, DMAP $(0.0391 \mathrm{~g}, 0.3 \mathrm{mmol})$, DCM $(6.5 \mathrm{~mL}), 20 \mathrm{~h}$. Yield = $72.4 \%$ (0.4761 g, $1.09 \mathrm{mmol}) .{ }^{1} \mathrm{H}$ NMR (400 MHz; $\mathrm{CDCl}_{3}$; TMS): $\delta 0.929$ (s, 12H), 
Asymmetric Electrochemical Polymerization in Cholesteric Liquid Crystalline Media: Effect of Isomeric Structures of Chiral Inducers Containing Bornyl Group Atsushi Matsumura, Fan Yang, Hiromasa Goto*

Synthetic Metals, 202, 157-164 (2015).

0.978 (s, 6H), 1.120-1.164 (dd, 2H, $J=3.2,14.4 \mathrm{~Hz}), 1.290-1.464(\mathrm{~m}, 5 \mathrm{H}), 1.745-1.836$ (m, 4H), 2.128-2.196 (m, 2H), 2.467-2.524 (m, 2H), 5.126-5.164 (m, 2H), 7.536-7.575 $(\mathrm{t}, 1 \mathrm{H}, J=8.0 \mathrm{~Hz}), 8.232-8.254(\mathrm{dd}, 2 \mathrm{H}, J=1.2,7.8 \mathrm{~Hz}), 8.740-8.748(\mathrm{t}, 1 \mathrm{H}, J=1.6$ $\mathrm{Hz}) .{ }^{13} \mathrm{C}$ NMR (100 MHz; $\mathrm{CDCl}_{3}$; TMS): $\delta 13.61,18.93,19.73,27.39,28.06,36.93$, $45.01,47.93,49.17,80.97,128.61,130.64,131.27,133.56,166.01$.

\section{Synthesis of di(1,7,7-trimethylbicyclo[2.2.1]hept-2-ol)phthalate (I-o)}

Quantity used: Phthalic anhydride $(0.2254 \mathrm{~g}, 1.5 \mathrm{mmol}), \mathrm{L}$-borneol (1.3917 g, 9.0 mmol), DCC (0.6250 g, $3.0 \mathrm{mmol})$, DMAP (0.0375 g, $0.3 \mathrm{mmol})$, DCM (5 mL), $20 \mathrm{~h}$. Yield $=94.6 \%(06227 \mathrm{~g}, 1.42 \mathrm{mmol}) .{ }^{1} \mathrm{H}$ NMR (400 MHz; $\left.\mathrm{CDCl}_{3} ; \mathrm{TMS}\right): \delta 0.898(\mathrm{~s}$, $3 \mathrm{H}), 0.906(\mathrm{~s}, 3 \mathrm{H}), 0.951(\mathrm{~s}, 3 \mathrm{H}), 1.184-1.228(\mathrm{dd}, 1 \mathrm{H}, J=3.4,13.6 \mathrm{~Hz}), 1.267-1.372$ $(\mathrm{m}, 2 \mathrm{H}), 1.714-1.796(\mathrm{~m}, 2 \mathrm{H}), 1.959-2.028(\mathrm{~m}, 1 \mathrm{H}), 2.418-2.497(\mathrm{~m}, 1 \mathrm{H}), 5.103-5.136$ (m, 1H), 7.521-7.544 (dd, 1H, $J=3.4,5.6 \mathrm{~Hz}), 7.710-7.733(\mathrm{dd}, 1 \mathrm{H}, J=3.4,5.6 \mathrm{~Hz}$ ). ${ }^{13} \mathrm{C}$ NMR (100 MHz; $\mathrm{CDCl}_{3}$; TMS): $\delta 13.57$ 18.89, 19.71, 27.30, 28.00, 36.40, 44.92, $47.93,48.98,81.39,128.92,130.76,132.93,167.68$.

\section{Synthesis of tri(1,7,7-trimethylbicyclo[2.2.1]hept-2-ol)-1,3,5-benzenetricarboxylate (I-t)}

Quantity used: Trimesic acid (0.3125 g, $1.5 \mathrm{mmol}), L$-borneol (1.3852 g, $9.0 \mathrm{mmol})$, DCC (0.9275 g, $4.5 \mathrm{mmol})$, DMAP (0.0530 g, $0.5 \mathrm{mmol})$, DCM (5 mL), $23 \mathrm{~h}$. Yield = $36.4 \%$ (0.3383 g, $0.55 \mathrm{mmol}) .{ }^{1} \mathrm{H}$ NMR (400 MHz; $\left.\mathrm{CDCl}_{3} ; \mathrm{TMS}\right): \delta 0.934(\mathrm{~s}, 6 \mathrm{H})$, 0.982 (s, 3H), 1.133-1.176 (dd, $1 \mathrm{H}, J=1.7,13.9 \mathrm{~Hz}), 1.298-1.363(\mathrm{~m}, 1 \mathrm{H}), 1.409-1.471$ $(\mathrm{m}, 1 \mathrm{H}), 1.756-1.863(\mathrm{~m}, 2 \mathrm{H}), 2.129-2.196(\mathrm{~m}, 1 \mathrm{H}), 2.480-2.537(\mathrm{~m}, 1 \mathrm{H}), 5.150-5.188$ $(\mathrm{m}, 1 \mathrm{H}), 8.886(\mathrm{~s}, 1 \mathrm{H}) .{ }^{13} \mathrm{C} \mathrm{NMR}\left(100 \mathrm{MHz} ; \mathrm{CDCl}_{3}\right.$; TMS $): \delta 13.61,18.93,19.73$, $27.39,28.04,36.92,45.00,47.96,49.21,81.43,131.84,134.29,165.25$.

\section{Polymerization}

A CLC electrolyte consisted of the chiral inducer, TBAP, a monomer and 6CB. The CLC electrolyte solution was injected between two indium tin oxide (ITO) glass electrodes sandwiching a Teflon sheet (thickness $0.2 \mathrm{~mm}$ ) as a spacer. Polymerization was performed with constant $4.0 \mathrm{~V}$ direct-current voltage. During polymerization, temperature was being held at $15{ }^{\circ} \mathrm{C}$ in order to maintain the CLC phase. After $5 \mathrm{~min}$, a thin polymer film was deposited on the anode side of the ITO glass electrode. The residual CLC solution was washed off with $n$ - hexane to obtain a pure polymer film.

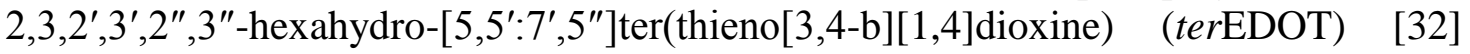
was used as a monomer for the asymmetric electrochemical polymerization. The terEDOT was prepared by previously reported method [16].

\section{Results and Discussion}

\section{Synthesis of chiral inducers}

The five chiral inducers were synthesized from aromatic carboxylic acid and $L$ borneol by condensation reaction with dicyclohexyl carbodiimide (DCC) and dimethylaminopyridine (DMAP) (Scheme 1). Figure 1 shows IR spectra of the chiral inducers, showing clear absorption bands at around $700 \mathrm{~cm}^{-1}, 1200 \mathrm{~cm}^{-1}, 1700 \mathrm{~cm}^{-1}$ and 2900-2950 $\mathrm{cm}^{-1}$, derived from $\mathrm{C}-\mathrm{H}$ bending vibration of benzene ring, C-O-C stretching, $\mathrm{C}=\mathrm{O}$ stretching and $\mathrm{C}-\mathrm{H}$ stretching of the alkane in the bornyl group, 
Asymmetric Electrochemical Polymerization in Cholesteric Liquid Crystalline Media: Effect of Isomeric Structures of Chiral Inducers Containing Bornyl Group

Atsushi Matsumura, Fan Yang, Hiromasa Goto*

Synthetic Metals, 202, 157-164 (2015).

respectively. These spectra indicate that the target compounds were successfully synthesized.

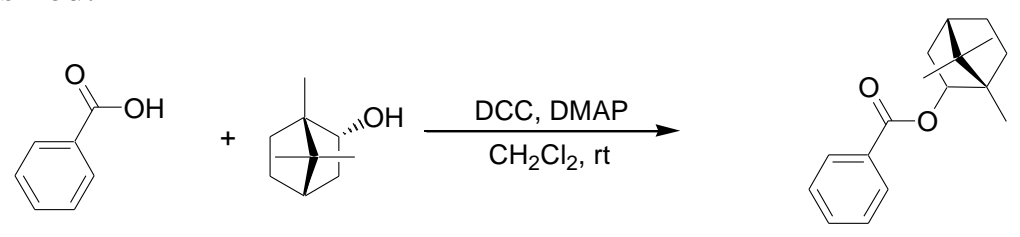

I-s, $48.7 \%$

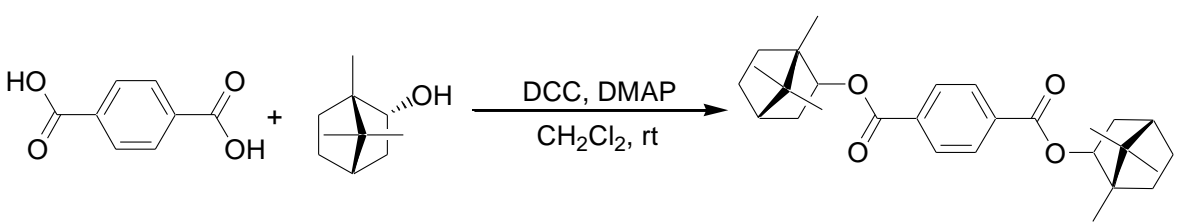

I-p, $63.0 \%$

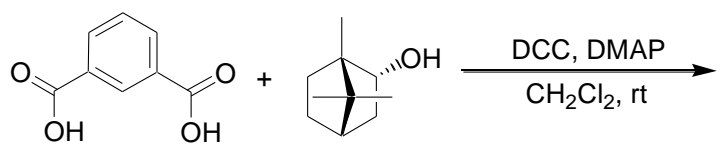<smiles>CC1(C)C2CC(OC(=O)c3cccc(C(=O)OC4C5CCC4(C)C5(C)C)c3)C1C2</smiles>

I-m, $72.4 \%$

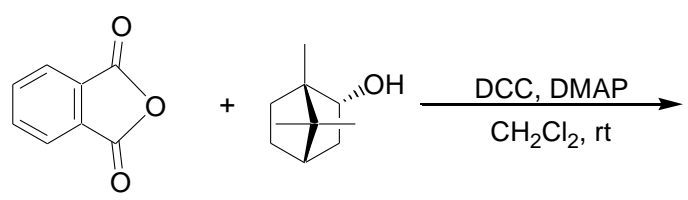<smiles>CC12C3CC(C3)CC1C2OC(=O)c1ccccc1C(=O)OCC12C3CC(C3)CC1C2(C)C</smiles>

I-o, $94.6 \%$

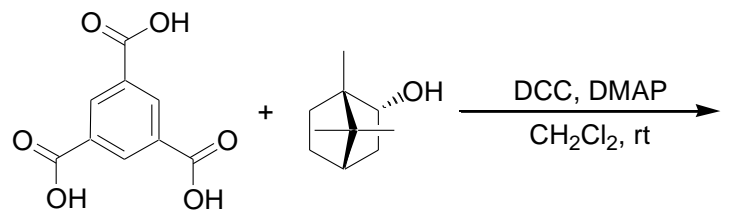<smiles>CC12CCC(C1)C1(C)CC2CC1(OC(=O)c1cc(C(=O)OC2CC3CCC2(C)C3)cc(C(=O)OC2CC3CCC2(C)C3(C)C)c1)C1CC2CCC1(C)C2</smiles>

I-t, $36.4 \%$

DCC : $N, N$ '-dicyclohexylcarbodiimide DMAP : $N, N$-dimethyl-4-aminopyridine

Scheme 1. Synthetic routes to chiral inducers containing bornyl group. 
Asymmetric Electrochemical Polymerization in Cholesteric Liquid Crystalline Media: Effect of Isomeric Structures of Chiral Inducers Containing Bornyl Group Atsushi Matsumura, Fan Yang, Hiromasa Goto*

Synthetic Metals, 202, 157-164 (2015).

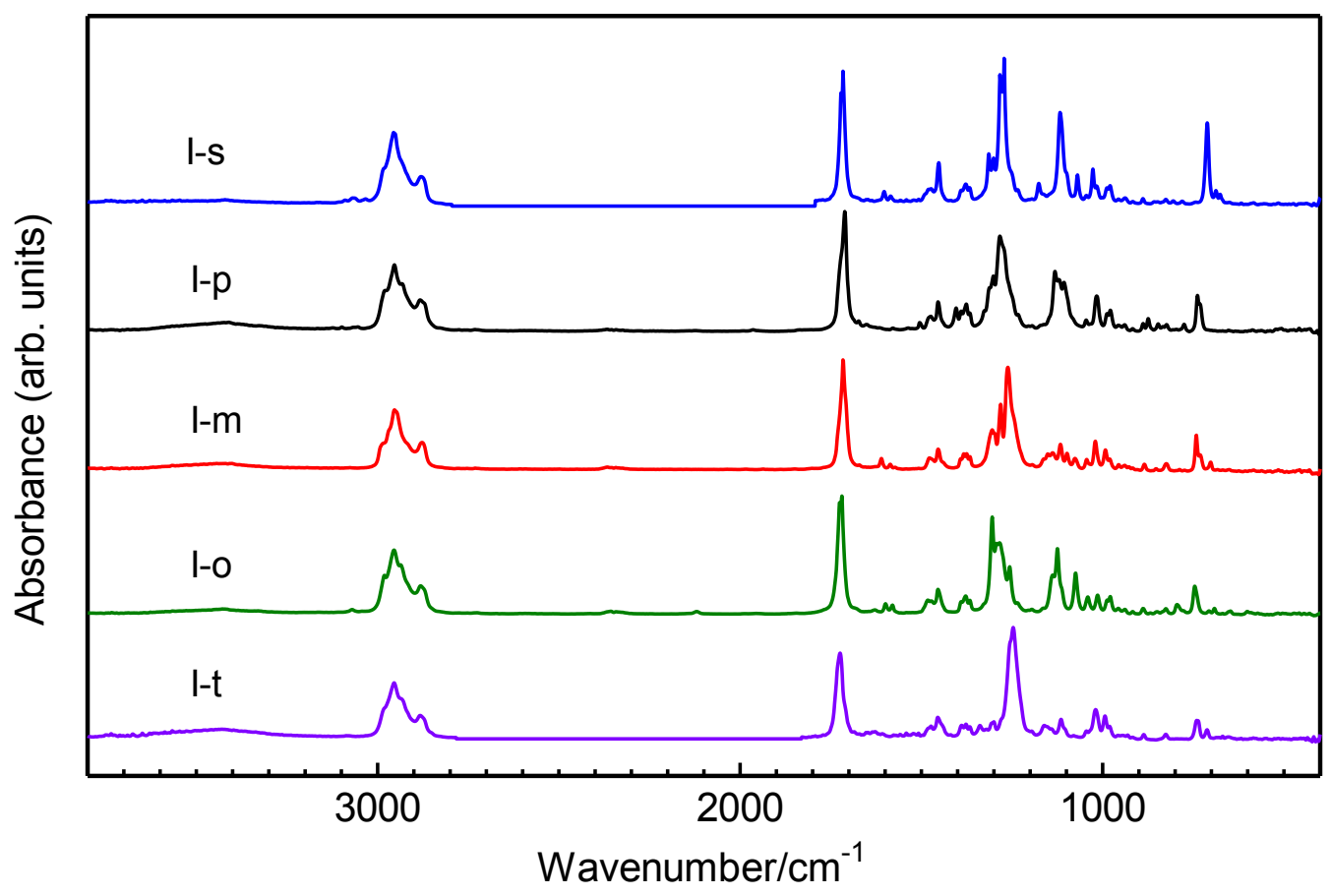

Fig. 1. IR spectra of the chiral inducers.

\section{Properties of the chiral inducers}

Table 1 shows helical twisting power (HTP), molar helical twisting power (MHTP) and macroscopic helical twisting power $\left(\beta_{M}\right)$ of the chiral inducers. HTP, MHTP and $\beta_{M}$ of the chiral inducers were examined by the Grandjean-Cano wedge method [33]. We calculated the value of these indexes:

$\mathrm{HTP}=(\mathrm{p} \cdot \mathrm{c})^{-1}$

$\mathrm{MHTP}=\mathrm{HTP} \cdot \mathrm{M}_{\mathrm{d}} \times 10^{-3}$

$\beta_{\mathrm{M}}=\left(\mathrm{p} \cdot \mathrm{c} \cdot \mathrm{M}_{\mathrm{h}} / \mathrm{M}_{\mathrm{d}}\right)^{-1}$.

Here, $\mathrm{p}$ and $\mathrm{c}$ are helical pitch of CLC and weight concentration of the chiral inducer, respectively; $M_{d}$ and $M_{h}$ are the molecular weight of the chiral inducer and molecular weight of the solvent, respectively. The $\beta_{\mathrm{M}}$ of I-p and I-m are larger than that of I-s. The increment of the $\beta_{M}$ suggests that increase of introduction number of bornyl groups in the chiral inducers strengthened the twisting ability. However, the $\beta_{M}$ of I-t is smaller than those of I-p and I-m. According to a previous report, twisting ability increases with a decrease of the flexible terminal alkyl chain length of chiral inducer [19]. Hence, the decrease of $\beta_{M}$ in the case of I-t can be due to an excess amount of bornyl groups. These results indicate that balance between the number of alkyl carbons and chiral centers determines twisting ability of the chiral inducers. Consequently, I-p and I-m show relatively high $\beta_{\mathrm{M}}$ due to good combination of the number of alkyl carbons and chiral centers. On the other hand, I-o shows the smallest $\beta_{\mathrm{M}}$ among them, although I-p and I-m have the same number of bornyl group. This obvious difference in $\beta_{M}$ can be explained by molecular affinity of chiral inducers with host liquid crystals. Twisting ability 
Asymmetric Electrochemical Polymerization in Cholesteric Liquid Crystalline Media: Effect of Isomeric Structures of Chiral Inducers Containing Bornyl Group

Atsushi Matsumura, Fan Yang, Hiromasa Goto*

Synthetic Metals, 202, 157-164 (2015).

generally relates to molecular shape of chiral inducers [26,34,35]. Thus, similarity of molecular geometry among chiral inducers and host liquid crystals is one of the important factors in modifying the twisting ability of the chiral inducers [28]. In this case, both 6CB and I-p have rod-like structures while I-o has V-shaped. Geometric incompatibility between a bent-shape I-o and a straight-shape $6 \mathrm{CB}$ makes $\beta_{\mathrm{M}}$ relatively smaller.

Optical properties were examined to investigate conformation of the chiral inducers in the solution state. Figure 2 shows the CD and the UV-vis absorption spectra of the chiral inducers in $0.1 \mathrm{mM} n$-hexane and acetonitrile solution. Table 2 summarizes the maximum absorption wavelengths $\left(\lambda_{\max }\right)$ and molar extinction coefficients $(\varepsilon)$. The chiral inducers show a relatively weak absorption band at $280 \mathrm{~nm}$ compared to each maximum absorption band. These weak absorption bands are due to $n-\pi^{*}$ transition of carbonyl groups [36]. The chiral inducers show maximum absorption wavelength at around 242 and $203 \mathrm{~nm}$. All of molar extinction coefficients of the chiral inducers at the maximum absorption are greater than $10^{4} \mathrm{M}^{-1} \mathrm{~cm}^{-1}$. I-o shows another absorption band at $225 \mathrm{~nm}$. The molar extinction coefficient of I-o at $225 \mathrm{~nm}$ is almost $10^{4} \mathrm{M}^{-1} \mathrm{~cm}^{-1}$. Such large values of the molar extinction coefficients indicate that the maximum absorption bands are due to allowed transition. Therefore, these absorption properties demonstrate that the maximum absorption bands derive from $\pi-\pi^{*}$ transition.

I-o shows characteristic optical properties compared to the other chiral inducers. The value of molar extinction coefficient at $225 \mathrm{~nm}$ of I-o in acetonitrile solution is smaller than that in $n$-hexane solution. In the other absorption bands due to $\pi-\pi^{*}$ transitions, however, the values of molar extinction coefficients in acetonitrile solution are greater than that in $n$-hexane solution. In the CD spectra, only I-o shows a CD signal at $243 \mathrm{~nm}$ in acetonitrile solution. The $\mathrm{CD}$ signal and small molar extinction coefficients in acetonitrile solution suggests a distorted $\pi$-system of the I-o in acetonitrile solution. To investigate the conformation of $\mathrm{I}-\mathrm{O}$ in acetonitrile solution, the concentration dependence of optical properties of I-o in acetonitrile solution was further examined. Figure 3 shows the $\mathrm{CD}$ and the UV-vis absorption spectra of I-o in acetonitrile solution at various concentrations. Values of molar extinction coefficients decreased and maximum absorption wavelength red-shifted with increasing concentration of solution. This concentration dependence is due to hypochromic effect and indicates that I-o forms aggregation in acetonitrile solution. The red-shift also supports formation of the aggregation. However, no concentration dependence is observable in the CD spectra, meaning that the CD signal at $243 \mathrm{~nm}$ is independent of aggregation of I-o. Hence, we consider that the $\mathrm{CD}$ signal of I-o derives from distorted $\pi$ system induced by polar solvent. Since $6 \mathrm{CB}$ is also polar solvent, I-o may have distorted structure in 6CB solution. When a chiral inducer has chiral aliphatic centers, short range solute-solvent interaction modulated by the solute molecular shape should be more important than polarity and electrostatic properties [37]. In this case, distortion of the $\pi$-system mainly affected for the twisting ability. Therefore, the weak twisting ability of I-o may be derived from a distorted $\pi$-system. 
Asymmetric Electrochemical Polymerization in Cholesteric Liquid Crystalline Media: Effect of Isomeric Structures of Chiral Inducers Containing Bornyl Group

Atsushi Matsumura, Fan Yang, Hiromasa Goto*

Synthetic Metals, 202, 157-164 (2015).

Table 1. Helical twisting power of the chiral inducers.

\begin{tabular}{lcccc}
\hline Chiral inducer & $\begin{array}{c}\mathrm{LC} \\
\text { solvent }\end{array}$ & $\begin{array}{c}\mathrm{HTP}^{\mathrm{b}} \\
\left(\mu \mathrm{m}^{-1}\right)\end{array}$ & $\begin{array}{c}\mathrm{MHTP}^{\mathrm{c}} \\
\left(\mu \mathrm{m}^{-1} \mathrm{~mol}^{-1} \mathrm{~kg}\right)\end{array}$ & $\begin{array}{c}\beta_{\mathrm{M}}{ }^{\mathrm{d}} \\
\left(\mu \mathrm{m}^{-1}\right)\end{array}$ \\
\hline I-s & $6 \mathrm{CB}^{\mathrm{a}}$ & 6.95 & 1.80 & 6.82 \\
I-p & $6 \mathrm{CB}$ & 9.92 & 4.35 & 16.52 \\
I-m & $6 \mathrm{CB}$ & 8.69 & 3.81 & 14.46 \\
I-o & $6 \mathrm{CB}$ & 2.27 & 0.99 & 3.77 \\
I-t & $6 \mathrm{CB}$ & 5.76 & 3.56 & 13.53 \\
\hline
\end{tabular}

a 4-Cyano-4'- $n$-hexyl biphenyl.

${ }^{\mathrm{b}} \mathrm{HTP}=(\mathrm{p} \cdot \mathrm{c})^{-1} ; \mathrm{p}=$ helical pitch; $\mathrm{c}=$ weight concentration of the chiral inducer.

${ }^{c}$ MHTP (molar helical twisting power) $=\mathrm{HTP} \cdot \mathrm{M}_{\mathrm{d}} \times 10^{-3}, \mathrm{M}_{\mathrm{d}}=$ molecular weight of the chiral inducer.

${ }^{d} \beta_{M}=\left(p \cdot c \cdot M_{h} / M_{d}\right)^{-1} ; M_{h}=$ molecular weight of the solvent.

a)

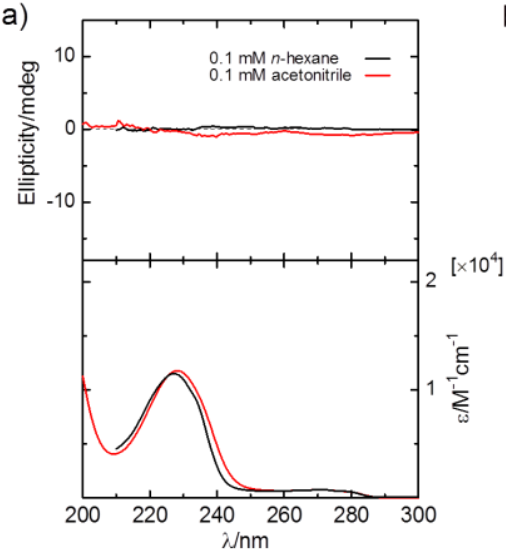

d)

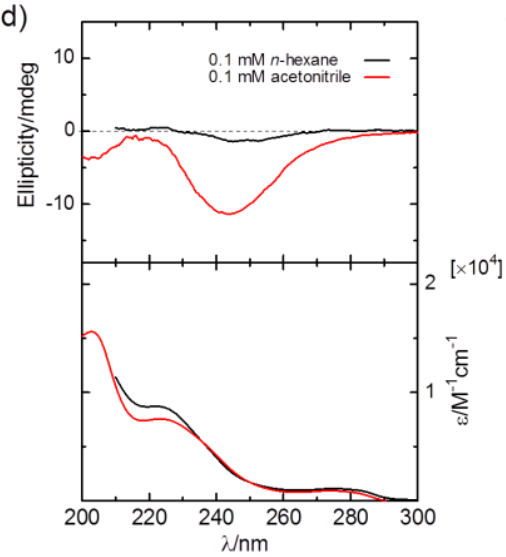

b)

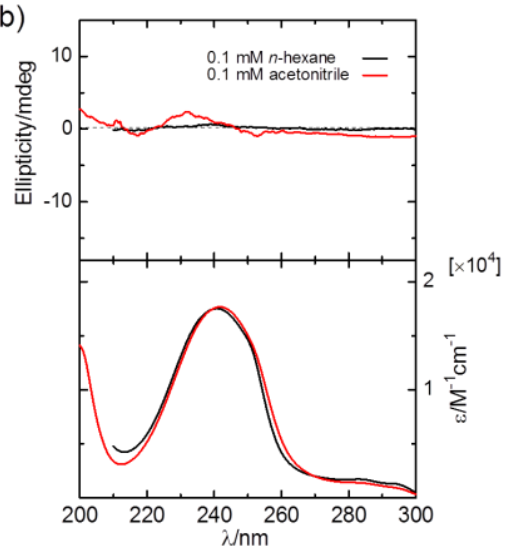

e)

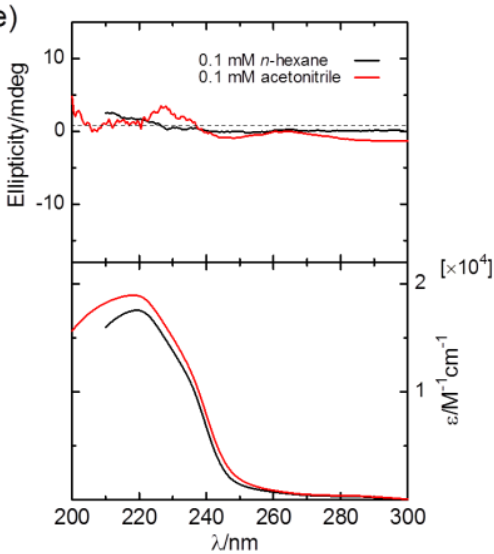

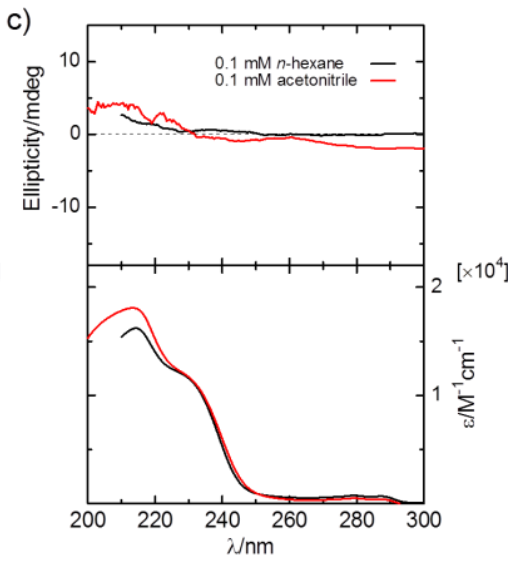

c)

Fig. 2. CD (upper) and UV-vis absorption (lower) spectra of (a) I-s, (b) I-p, (c) I-m, (d) I-o and (e) I-t in $0.1 \mathrm{mM} n$-hexane solution (black) and acetonitrile solution (red). 
Asymmetric Electrochemical Polymerization in Cholesteric Liquid Crystalline Media: Effect of Isomeric Structures of Chiral Inducers Containing Bornyl Group

Atsushi Matsumura, Fan Yang, Hiromasa Goto*

Synthetic Metals, 202, 157-164 (2015).

Table 2. UV-vis maximum absorption wavelengths and molar extinction coefficients of the chiral inducers.

\begin{tabular}{ccccc}
\hline Chiral inducer & $\begin{array}{c}\lambda_{\text {max, hexane }}{ }^{\mathrm{a}} \\
(\mathrm{nm})\end{array}$ & $\begin{array}{c}\lambda_{\max , \mathrm{ACN}}^{\mathrm{b}} \\
(\mathrm{nm})\end{array}$ & $\begin{array}{c}\varepsilon_{\text {hexane }}{ }^{\mathrm{c}} \\
\left(\mathrm{M}^{-1} \mathrm{~cm}^{-1} \times 10^{4}\right)\end{array}$ & $\begin{array}{c}\varepsilon_{\mathrm{ACN}}{ }^{\mathrm{d}} \\
\left(\mathrm{M}^{-1} \mathrm{~cm}^{-1} \times 10^{4}\right)\end{array}$ \\
\hline I-s & 227 & 228 & 1.15 & 1.18 \\
I-p & 241 & 242 & 1.76 & 1.78 \\
I-m & 215 & 214 & 1.63 & 1.81 \\
I-o & & 203 & & 1.56 \\
I-t & 220 & 218 & 1.76 & 1.90 \\
\hline
\end{tabular}

${ }^{\text {a }}$ Maximum absorption wavelength in $n$-hexane solution.

${ }^{\mathrm{b}}$ Maximum absorption wavelength in acetonitrile solution.

${ }^{\mathrm{c}}$ Molar extinction coefficient in $n$-hexane solution.

${ }^{\mathrm{d}}$ Molar extinction coefficient in acetonitrile solution.

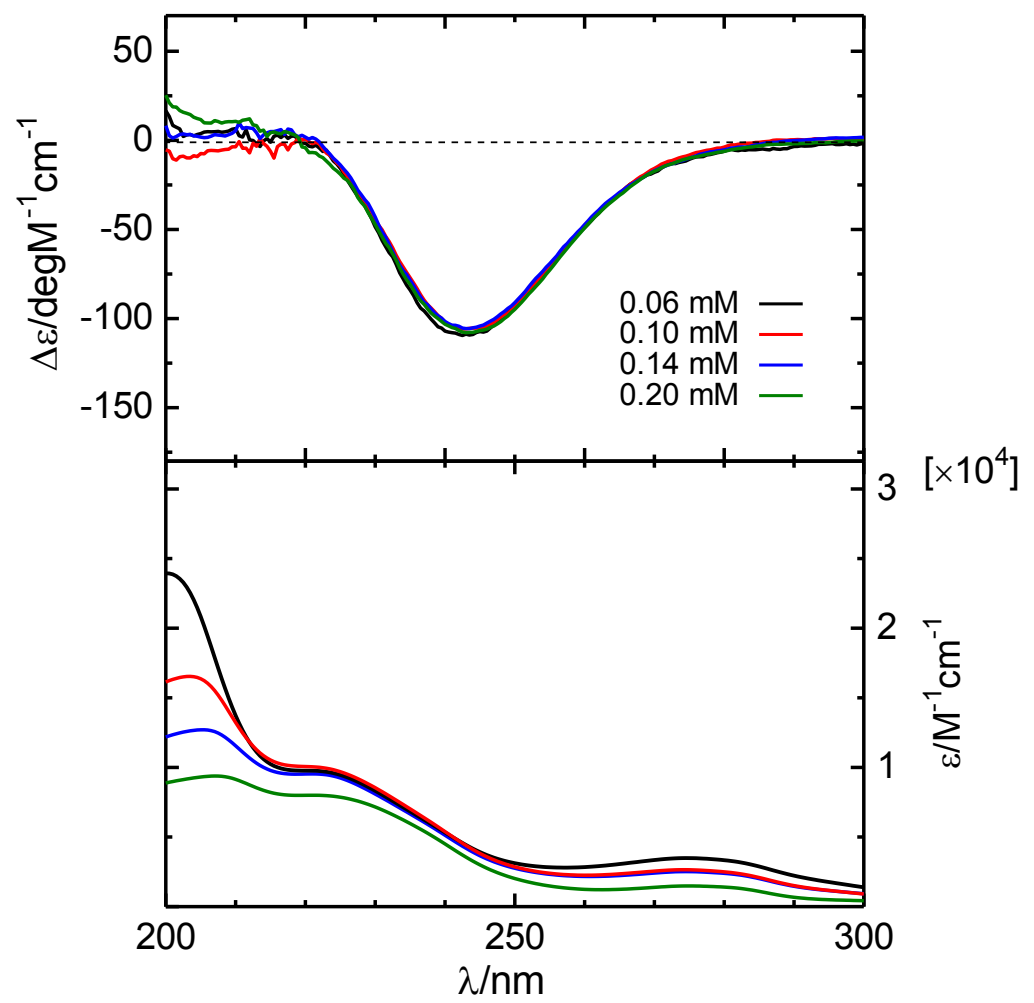

Fig. 3. CD (upper) and UV-vis absorption (lower) spectra of I-o in various concentration in acetonitrile solution.

\section{Properties of the polymer films}

Optically active poly(3,4-ethylenedioxythiophene) (PEDOT) films were prepared by asymmetric electrochemical polymerization. The composition of CLC electrolytes for this polymerization is summarized in Table 3. Scheme 2 shows polymerization conditions for preparation of the polymer films. Five polymer films were synthesized with I-s, I-p, I-m, I-o and I-t for construction of CLC electrolyte solution, and resultant 
Asymmetric Electrochemical Polymerization in Cholesteric Liquid Crystalline Media: Effect of Isomeric Structures of Chiral Inducers Containing Bornyl Group

Atsushi Matsumura, Fan Yang, Hiromasa Goto*

Synthetic Metals, 202, 157-164 (2015).

polymers are abbreviated as PF-single, PF-para, PF-meta, PF-ortho and PF-triple, respectively.

Figure 4 shows $\mathrm{CD}$ and UV-vis absorption spectra. The polymer films show first positive and second negative Cotton effect corresponding to maximum absorption wavelength in the UV-vis $\left(\lambda_{\max }\right)$. This type of bisignate Cotton effect is due to right-handed exciton coupling of polymer backbones via Davydov splitting. The CD spectra indicate that the PEDOT backbones form right-handed helical aggregation. Figure 5 shows plots of ellipticities at first positive and second negative CD signal vs. HTP of the chiral inducers. Degree of the ellipticity of the polymer films is almost proportional to the HTP of the chiral inducers. This relationship suggests that formation of helical aggregation strongly depends on the twisting ability of the chiral inducer. In UV-vis absorption spectra, PF-para shows largest $\lambda_{\max }$ in the polymer films synthesized in this study. Moreover, in both CD and UV-vis absorption spectra, PF-para shows the broadest spectra in the all polymer films. In conjugated polymer, maximum absorption wavelengths are red-shifted and absorption spectra are broadened due to intermolecular $\pi-\pi$ stacking in film state. Hence, the spectroscopic properties of PF-para indicate that I-p induced tight $\pi-\pi$ stacking of PEDOT during the polymerization. Figure 6 shows POM images of the polymer films. Vortex structure was observed. These structures are similar to a typical finger print texture of a cholesteric phase. The $\mathrm{CD}$ results and the vortex structures indicate that the direction of polymer chains is arranged by orientation of CLC. As shown in Figure 6, size of voltex sructure in PF-ortho is larger than those of the other polymer films. This suggests that helical pitch of polymer film depends on HTP of the chiral inducer [19].

Figure 7 shows CD and UV-vis absorption spectra of PF-para at various applied potentials against an $\mathrm{Ag} / \mathrm{Ag}^{+}$reference electrode during the oxidation process. The UV-vis maximum absorption intensities gradually decrease while broad absorption bands at long wavelengths appear. In the CD spectra, first positive CD signal decrease and second negative CD signal increase with increasing applied voltage. The changes are due to generation of polarons (radical cationic states) in electrochemical doping. Polymer main chains have quinonoid structure in polarons. On the other hand, polymers in reduced state consist of benzenoid structure. Quinonoid structure improves coplanarity of polymer backbones in comparison with benzenoid structure. The difference of coplanarity and conformation between polarons and reduced state cause the changes in the UV-vis absorption and the CD spectra. Figure 8 shows ORD spectra of PF-para at various applied potentials against an $\mathrm{Ag} / \mathrm{Ag}^{+}$reference electrode in oxidation process. The ORD spectra show clear isosbestic point at $584 \mathrm{~nm}$. The existence of an isosbestic point means that only the reduced state and polarons exist in PF-para during oxidation. 
Asymmetric Electrochemical Polymerization in Cholesteric Liquid Crystalline Media: Effect of Isomeric Structures of Chiral Inducers Containing Bornyl Group

Atsushi Matsumura, Fan Yang, Hiromasa Goto*

Synthetic Metals, 202, 157-164 (2015).

Table 3. Constituents of cholesteric liquid crystal electrolyte solution.

\begin{tabular}{|c|c|c|}
\hline Reagent & $\begin{array}{l}\text { Chemical } \\
\text { structure }\end{array}$ & wt $\%$ \\
\hline $6 \mathrm{CB}$ & $\mathrm{C}_{6} \mathrm{H}_{13}$ & $94.2 \%$ \\
\hline Chiral inducer & $\begin{array}{c}\text { Chiral inducer } \\
\text { containing bornyl } \\
\text { group }\end{array}$ & $2.8 \%$ \\
\hline Monomer & & $2.8 \%$ \\
\hline $\begin{array}{c}\text { TBAP } \\
\text { (Supporting salt) }\end{array}$ & & $0.2 \%$ \\
\hline
\end{tabular}

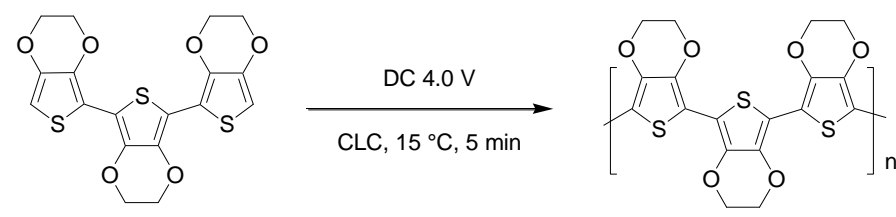

Scheme 2. Electrochemical synthesis of poly(3,4-ethylenedioxythiophene) (PEDOT). 
Asymmetric Electrochemical Polymerization in Cholesteric Liquid Crystalline Media: Effect of Isomeric Structures of Chiral Inducers Containing Bornyl Group Atsushi Matsumura, Fan Yang, Hiromasa Goto*

Synthetic Metals, 202, 157-164 (2015).

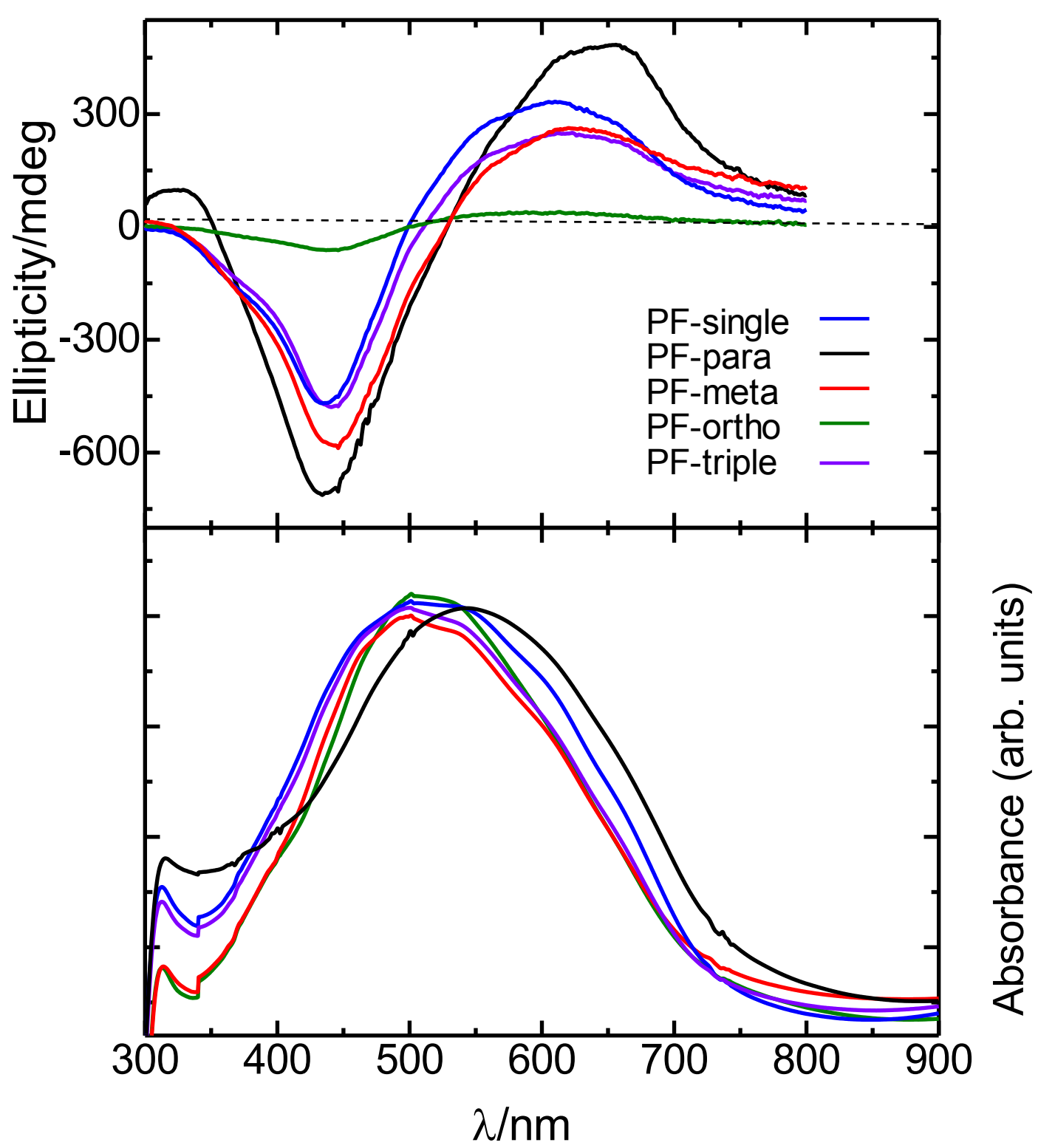

Fig. 4. CD (upper) and UV-vis absorption (lower) spectra. 
Asymmetric Electrochemical Polymerization in Cholesteric Liquid Crystalline Media: Effect of Isomeric Structures of Chiral Inducers Containing Bornyl Group

Atsushi Matsumura, Fan Yang, Hiromasa Goto*

Synthetic Metals, 202, 157-164 (2015).

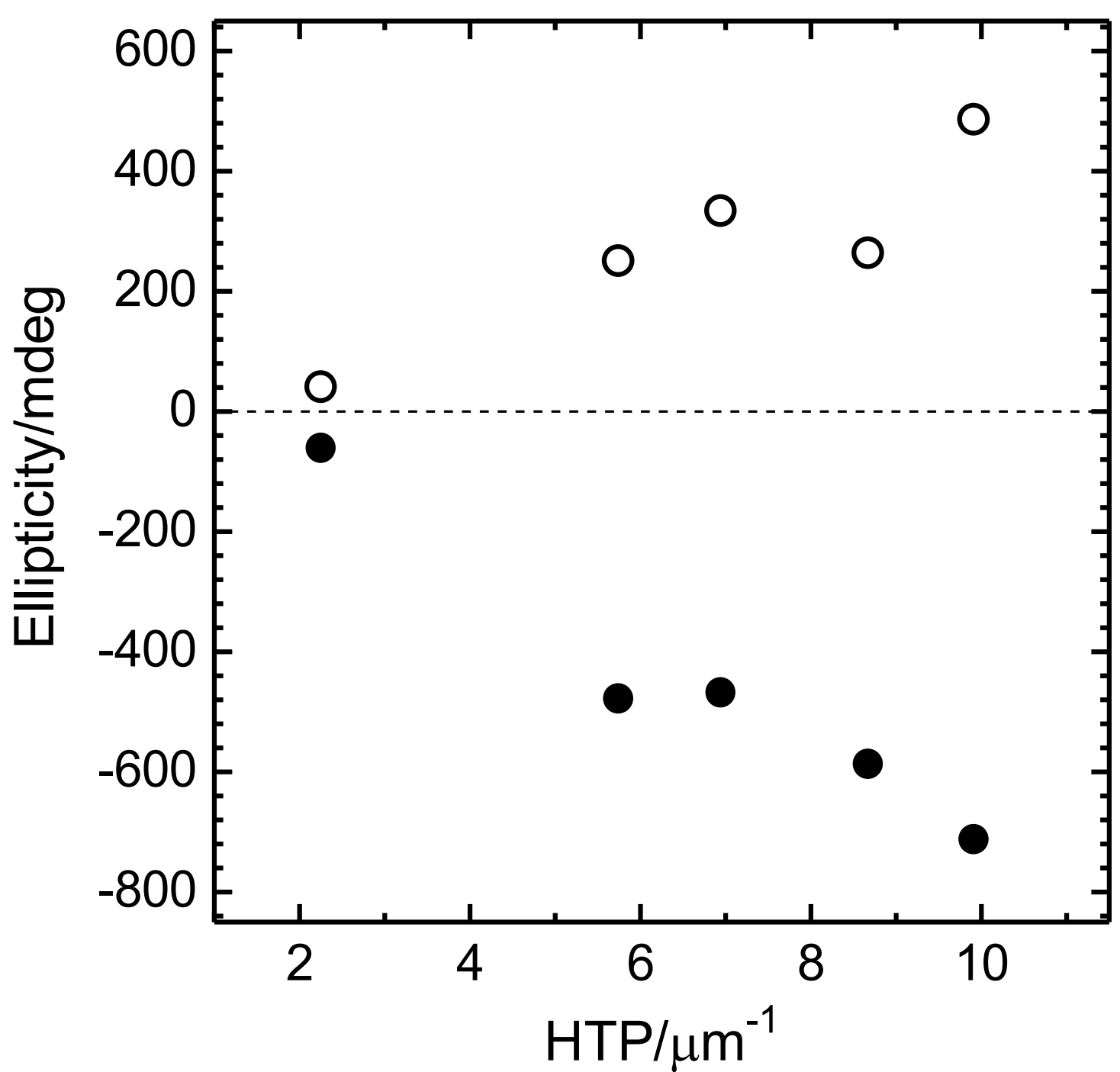

Fig. 5. Plots of positive (white) and negative (black) CD intensities of the polymer films vs. HTPs of the chiral inducers. 
Asymmetric Electrochemical Polymerization in Cholesteric Liquid Crystalline Media: Effect of Isomeric Structures of Chiral Inducers Containing Bornyl Group Atsushi Matsumura, Fan Yang, Hiromasa Goto*

Synthetic Metals, 202, 157-164 (2015).
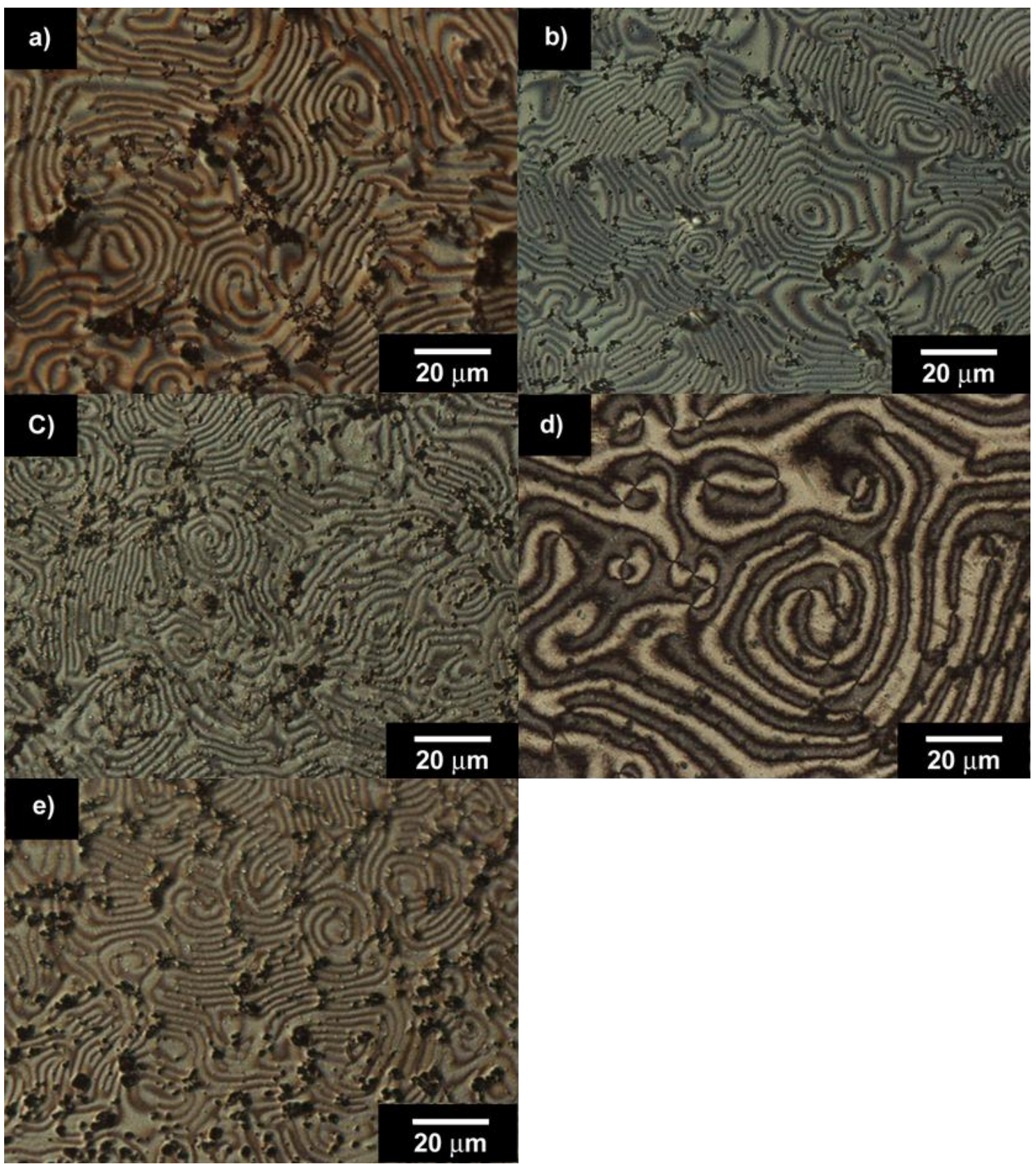

Fig. 6. Polarizing optical microscopy (POM) images of polymer films. (a) PF-single; (b) PF-para; (c) PF-meta; (d) PF-ortho; (e) PF-triple. 
Asymmetric Electrochemical Polymerization in Cholesteric Liquid Crystalline Media: Effect of Isomeric Structures of Chiral Inducers Containing Bornyl Group Atsushi Matsumura, Fan Yang, Hiromasa Goto*

Synthetic Metals, 202, 157-164 (2015).

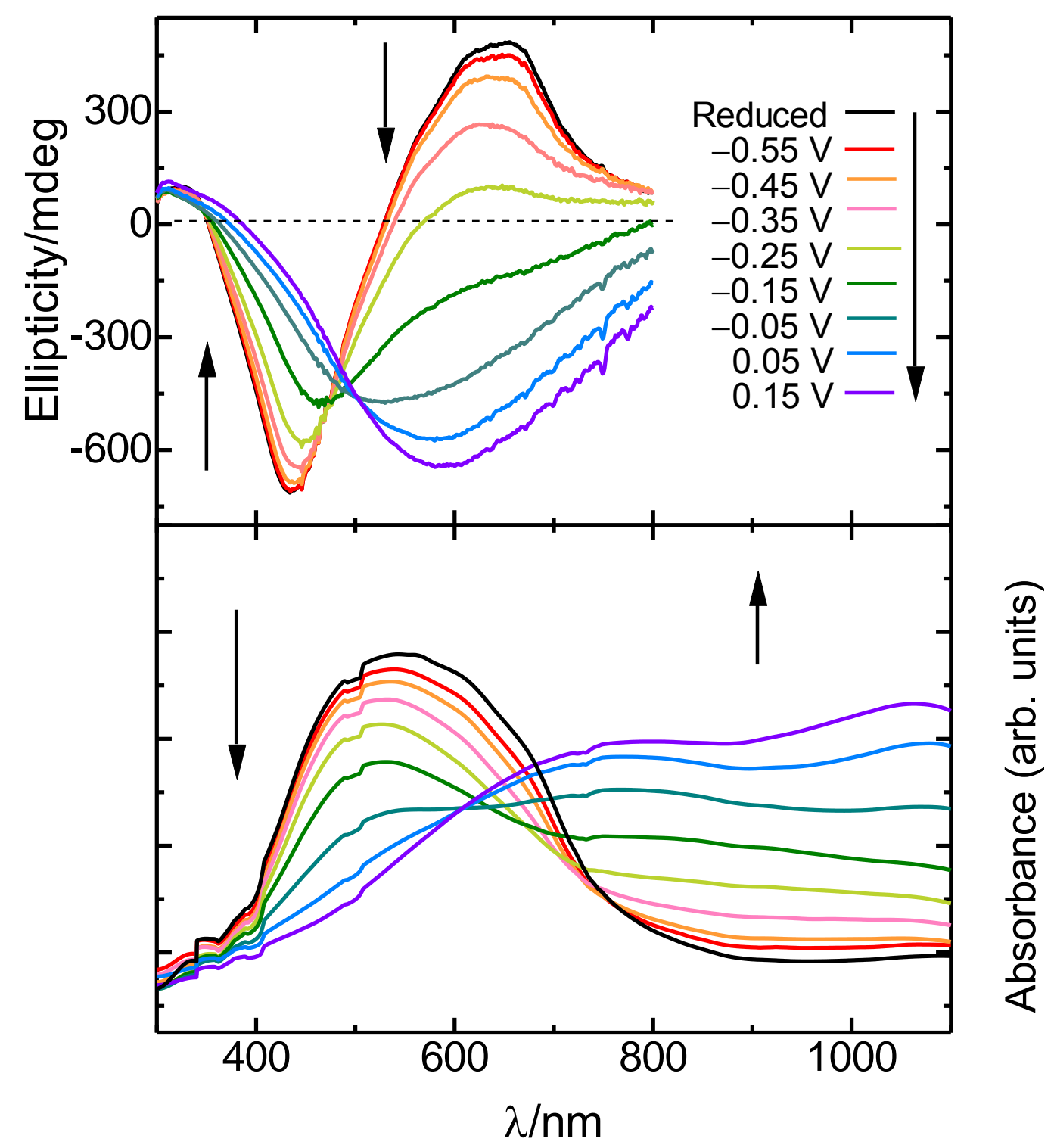

Fig. 7. CD (upper) and UV-vis absorption (lower) spectra of PF-para. 
Asymmetric Electrochemical Polymerization in Cholesteric Liquid Crystalline Media: Effect of Isomeric Structures of Chiral Inducers Containing Bornyl Group

Atsushi Matsumura, Fan Yang, Hiromasa Goto*

Synthetic Metals, 202, 157-164 (2015).

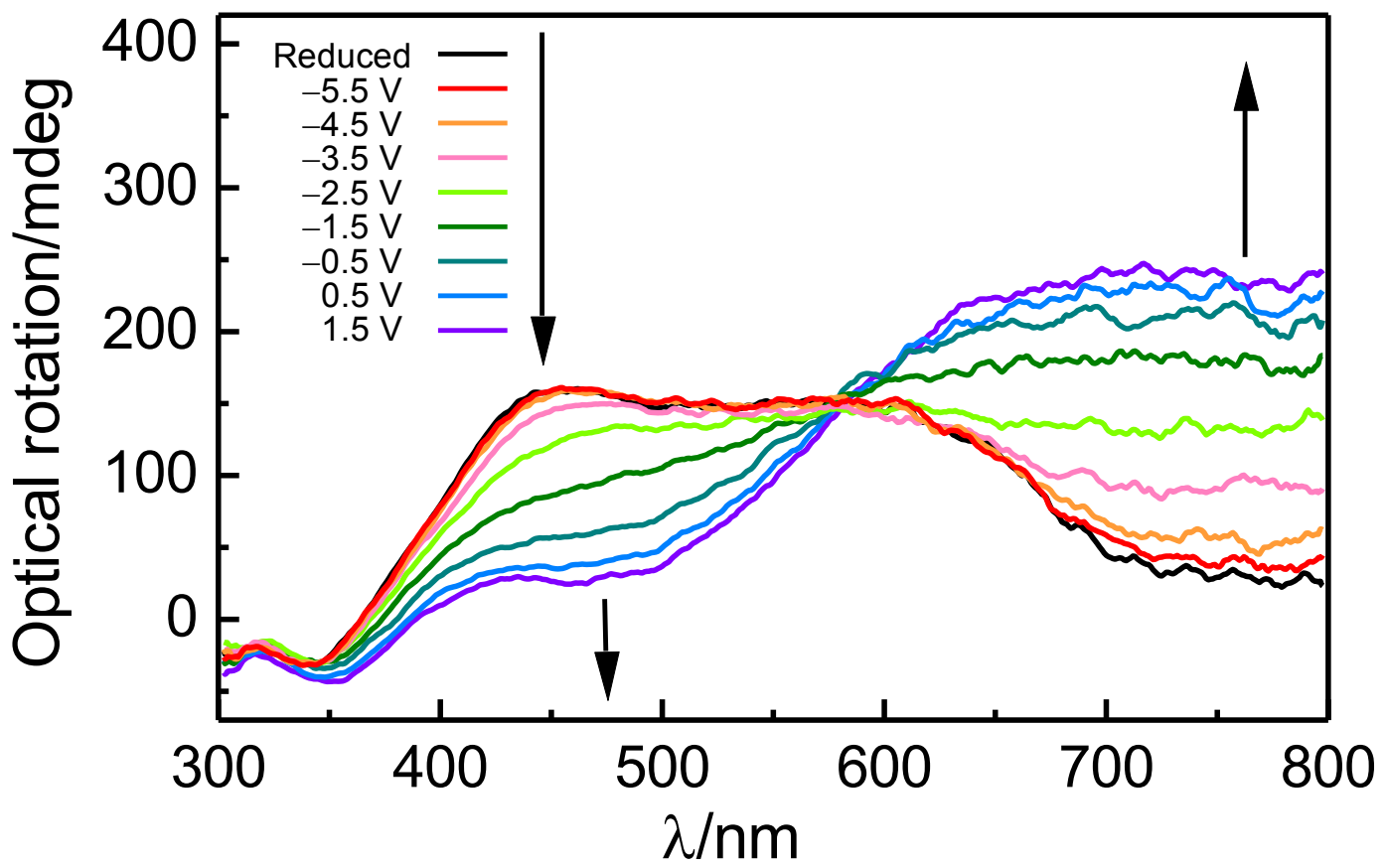

Fig. 8. ORD spectra of PF-para.

\section{Conclusions}

The chiral inducers with bornyl group having twisting ability were synthesized. I-p shows the highest twisting ability due to the rod-like structure. I-o has low twisting ability because of $V$-shaped structure and distortion of $\pi$-system. Optically active PEDOT films were prepared by asymmetric electrochemical polymerization using the chiral inducers. The PEDOT backbones form right-handed helical aggregation. The CD signal intensities of the PEDOT films are almost proportional to HTP of the chiral inducers. I-p induces tight $\pi$ stacking between PEDOT backbones in the polymerization. Electro-chiroptically active conjugated polymers are obtained with chirality transcription of Bornyl-based chiral compounds via LC form chiral structure imprint.

\section{References}

[1] C. Tang, K. Hackenberg, Q. Fu, P. M. Ajayan, and H. Ardebili, Nano Lett. 12, 1152 (2012).

[2] Z. Shen, L. Shi, B. You, L. Wu, and D. Zhao, J. Mater. Chem. 22, 8069 (2012).

[3] Y. Fujitsu, H. Nakayama, T. Uchida, S. Yamazaki, and K. Kimura, J. Polym. Sci. Part A Polym. Chem. 51, 780 (2013).

[4] R. Dattani, J. H. Bannock, Z. Fei, R. C. I. MacKenzie, A. A. Y. Guilbert, M. S. Vezie, J. Nelson, J. C. de Mello, M. Heeney, J. T. Cabral, and A. J. Nedoma, J. Mater. Chem. A 2, 14711 (2014).

[5] S. Vandeleene, M. Jivanescu, A. Stesmans, J. Cuppens, M. J. Van Bael, T. Verbiest, and G. Koeckelberghs, Macromolecules 44, 4911 (2011).

[6] I. Tahar-djebbar, F. Nekelson, B. Heinrich, B. Donnio, D. Guillon, D. Kreher, F. 
Asymmetric Electrochemical Polymerization in Cholesteric Liquid Crystalline Media: Effect of Isomeric Structures of Chiral Inducers Containing Bornyl Group Atsushi Matsumura, Fan Yang, Hiromasa Goto*

Synthetic Metals, 202, 157-164 (2015).

Mathevet, Chem. Mater. 23, 4653 (2011).

[7] Y. Kawagoe, M. Fujiki, and Y. Nakano, New J. Chem. 34, 637 (2010).

[8] J. Vogelsang, T. Adachi, J. Brazard, D. A. Vanden Bout, and P. F. Barbara, Nat. Mater. 10, 942 (2011).

[9] H. Dong, H. Li, E. Wang, Z. Wei, W. Xu, W. Hu, and S. Yan, Langmuir 24, 13241 (2008).

[10]C. Li, M. Numata, A.-H. Bae, K. Sakurai, and S. Shinkai, J. Am. Chem. Soc. 127, 4548 (2005).

[11]R. Österbacka, C. P. An, X. M. Jiang, Z. V. Vardeny, Science 287, 839 (2000).

[12] Y. Y. Zhu, T. T. Yin, J. Yin, N. Liu, Z. Yu, Y. Zhu, Y. Ding, J. Yin, Z. Q. Wu, RSC Adv. 4, 40241 (2014).

[13]A. Satrijo and T. M. Swager, Macromolecules 38, 4054 (2005).

[14]H. Goto, IOP Conf. Ser.: Mater. Sci. Eng. 54012013 (2014).

[15]H. Goto, J. Polym. Sci. Part A Polym. Chem. 45, 1377 (2007).

[16]H. Goto, J. Mater. Chem. 19, 4914 (2009).

[17]H. Yoneyama, A. Tsujimoto, and H. Goto, Macromolecules 40, 5279 (2007).

[18]K. Kawabata, M. Takeguchi, and H. Goto, Macromolecules 46, 2078 (2013).

[19]H. Hayashi, A. Wang, K. Kawabata, and H. Goto, Mater. Chem. Phys. 137, 816, (2013).

[20]D. J. Earl and M. R. Wilson, J. Chem. Phys. 119, 10280 (2003).

[21]H. Hayashi, K. Kawabata, and H. Goto, Int. Lett. Chem. Phys. Astron. 8, 6 (2014).

[22]T. Iseki, K. Kawabata, S. Nimori, and H. Goto, Synth. Met. 187, 217 (2014).

[23]P. E. McGovern, A. Mirzoian, and G. R. Hall, Proc. Natl. Acad. Sci. U. S. A. 106, 7361 (2009).

[24]L. Sun, L. Zhu, P. Lu, T. Yuen, J. Li, R. Ma, R. Baliram, S. S. Moonga, P. Liu, A. Zallone, M. I. New, T. F. Davies, M. Zaidi, R. Baliram, B. P. Luley, N. Rovira, A. Mirzoian, M. P. Callahan, K. E. Smith, and G. R. Hall, Proc. Natl. Acad. Sci. 110, 12498 (2013).

[25]F. J. Devlin, P. J. Stephens, and P. Besse, J. Org. Chem. 70, 2980 (2005).

[26]A. di Matteo, S. M. Todd, G. Gottarelli, G. Solladié, V. E. Williams, R. P. Lemieux, A. Ferrarini, and G. P. Spada, J. Am. Chem. Soc. 123, 7842 (2001).

[27]A. Januszko, P. Kaszynski, and W. Drzewinski, J. Mater. Chem. 16, 452 (2006).

[28]J. Liu and P. Yang, J. Appl. Polym. Sci., 91, 3693 (2004).

[29]J.H. Liu, H.J. Hung, D.S. Wu, S.M. Hong, and A. Y. G. Fu, J. Appl. Polym. Sci. 98, 88 (2005).

[30]C. Lazar, M. D. Wand, and R. P. Lemieux, J. Am. Chem. Soc. 122, 12586, (2000).

[31]A. Davidsson, B. Norden and S. Seth, Chem. Phys. Lett. 70, 313-316 (1980).

[32] J. M. Raimundo, P. Blanchard, H. Brisset, S. Akoudad, and J. Roncali, Chem. Commun. 939-940 (2000).

[33] Y. Wang, and Q. Li, Adv. Mater. 24, 1926-1945 (2012).

[34]A. Ferrarini, G. J. Moro, and P. L. Nordio, Phys. Rev. E 53, 681 (1996).

[35] S. Superchi, M. I. Donnoli, G. Proni, G. P. Spada, and C. Rosini, J. Org. Chem. 64, 4762 (1999).

[36]J. W. Sidman, Chem. Revs. 58, 689 (1958).

[37]S. Pieraccini, M. I. Donnoli, A. Ferrarini, G. Gottarelli, G. Licini, C. Rosini, S. Superchi, and G. P. Spada, J. Org. Chem. 68, 519 (2003). 
Asymmetric Electrochemical Polymerization in Cholesteric Liquid Crystalline Media: Effect of Isomeric Structures of Chiral Inducers Containing Bornyl Group

Atsushi Matsumura, Fan Yang, Hiromasa Goto*

Synthetic Metals, 202, 157-164 (2015).

Supplementary data for

Asymmetric Electrochemical Polymerization in Cholesteric Liquid Crystalline Media:

Effect of Isomeric Structures of Chiral Inducers Containing Bornyl Group

Atsushi Matsumura, Yang Fan, Hiromasa Goto*

Division of Materials Science, Faculty of Pure and Applied Sciences, University of

Tsukuba, Tsukuba, Ibaraki 305-8573, Japan

* Corresponding author. Tel.: +81-29-853-5128; fax: +81-29-853-4490; e-mail:

gotoh@ims.tsukuba.ac.jp 
Asymmetric Electrochemical Polymerization in Cholesteric Liquid Crystalline Media: Effect of Isomeric Structures of Chiral Inducers Containing Bornyl Group

Atsushi Matsumura, Fan Yang, Hiromasa Goto*

Synthetic Metals, 202, 157-164 (2015).

${ }^{1} \mathrm{H} \mathrm{NMR}\left(\mathrm{CDCl}_{3}, 400 \mathrm{MHz}\right)$

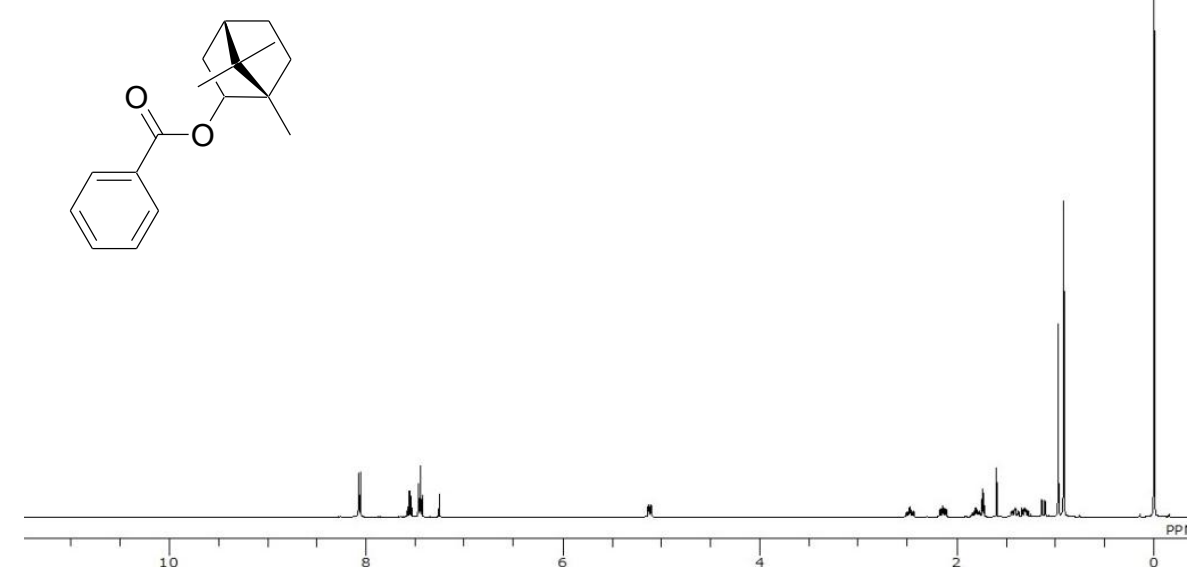

${ }^{13} \mathrm{C} \mathrm{NMR}\left(\mathrm{CDCl}_{3}, 100 \mathrm{MHz}\right)$

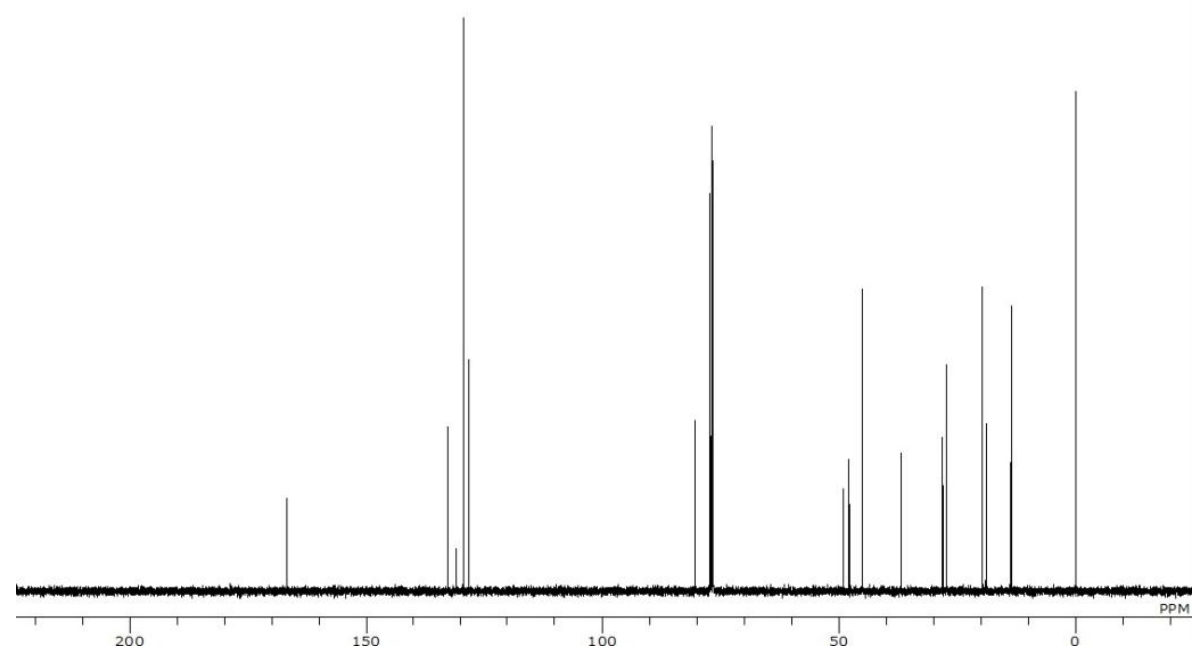

Fig. S1. ${ }^{1} \mathrm{H}$ (upper) and ${ }^{13} \mathrm{C}$ (lower) NMR spectra of I-s. 
Asymmetric Electrochemical Polymerization in Cholesteric Liquid Crystalline Media: Effect of Isomeric Structures of Chiral Inducers Containing Bornyl Group

Atsushi Matsumura, Fan Yang, Hiromasa Goto*

Synthetic Metals, 202, 157-164 (2015).
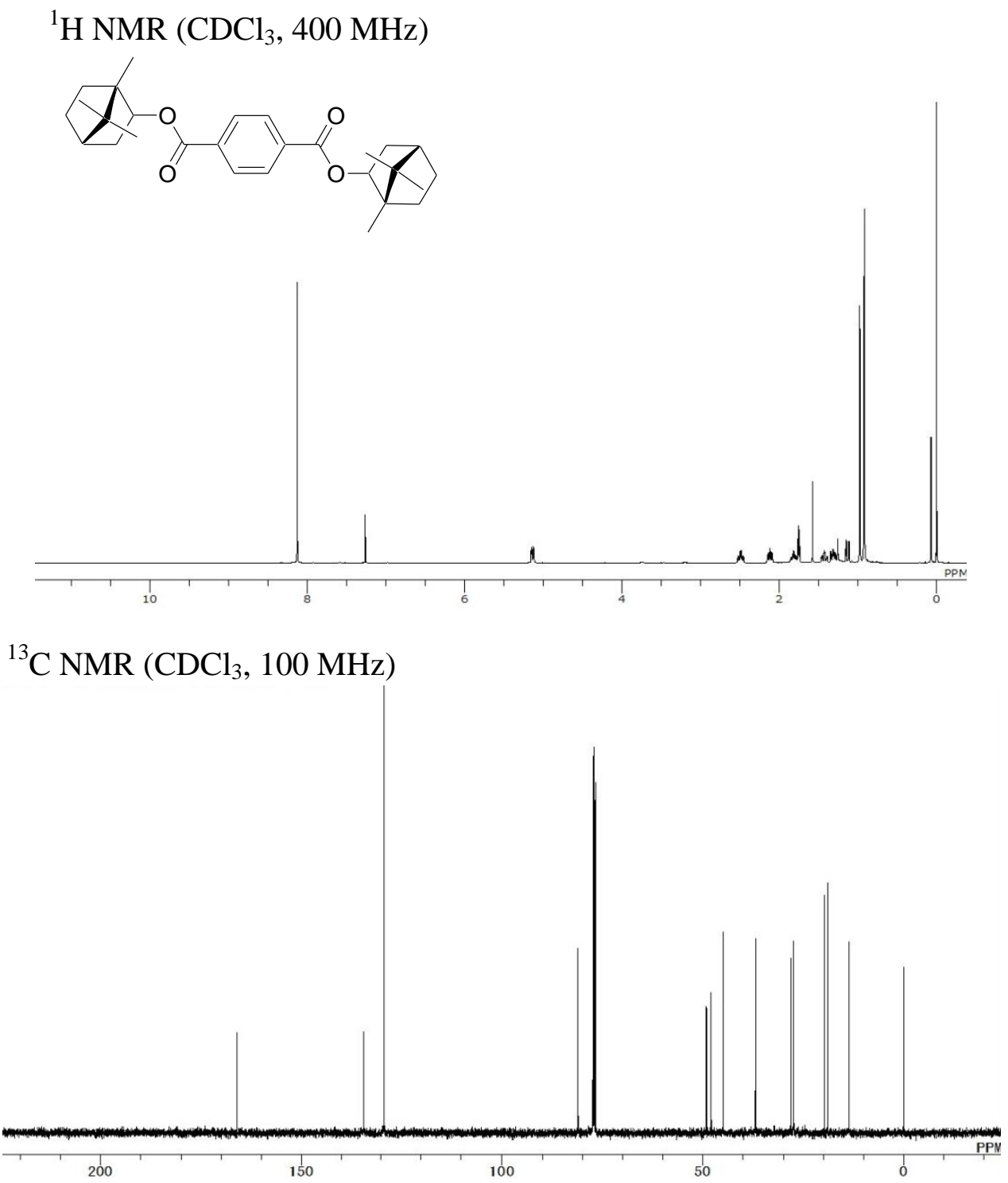

Fig. S2. ${ }^{1} \mathrm{H}$ (upper) and ${ }^{13} \mathrm{C}$ (lower) NMR spectra of I-p. 
Asymmetric Electrochemical Polymerization in Cholesteric Liquid Crystalline Media: Effect of Isomeric Structures of Chiral Inducers Containing Bornyl Group

Atsushi Matsumura, Fan Yang, Hiromasa Goto*

Synthetic Metals, 202, 157-164 (2015).

${ }^{1} \mathrm{H} \mathrm{NMR}\left(\mathrm{CDCl}_{3}, 400 \mathrm{MHz}\right)$

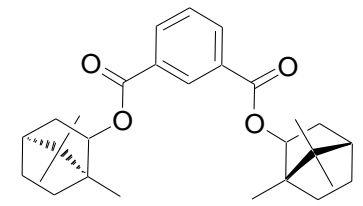

${ }^{13} \mathrm{C} \mathrm{NMR}\left(\mathrm{CDCl}_{3}, 100 \mathrm{MHz}\right)$

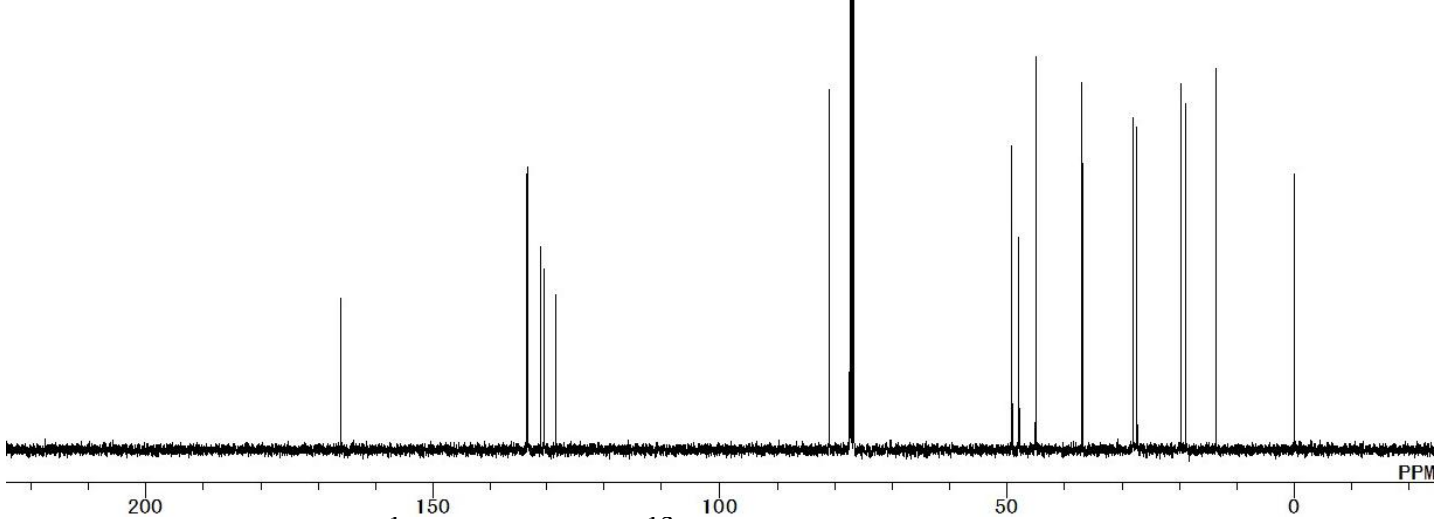

Fig. S3. ${ }^{1} \mathrm{H}$ (upper) and ${ }^{13} \mathrm{C}$ (lower) NMR spectra of I-m. 
Asymmetric Electrochemical Polymerization in Cholesteric Liquid Crystalline Media: Effect of Isomeric Structures of Chiral Inducers Containing Bornyl Group

Atsushi Matsumura, Fan Yang, Hiromasa Goto*

Synthetic Metals, 202, 157-164 (2015).

${ }^{1} \mathrm{H} \mathrm{NMR}\left(\mathrm{CDCl}_{3}, 400 \mathrm{MHz}\right)$
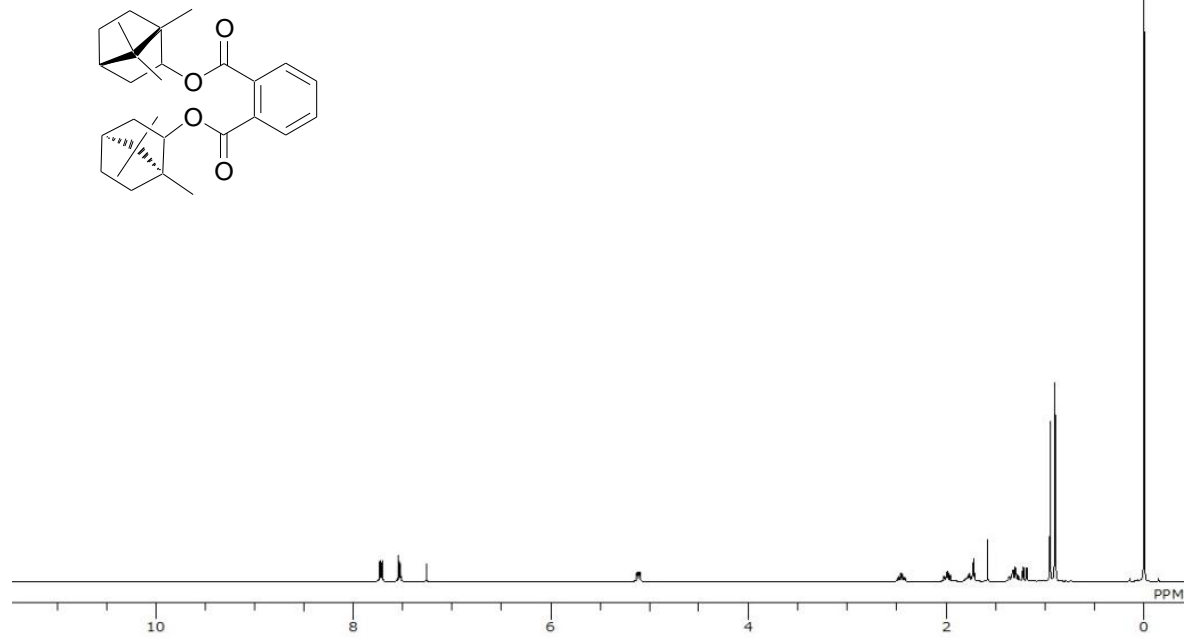

${ }^{13} \mathrm{C} \mathrm{NMR}\left(\mathrm{CDCl}_{3}, 100 \mathrm{MHz}\right)$

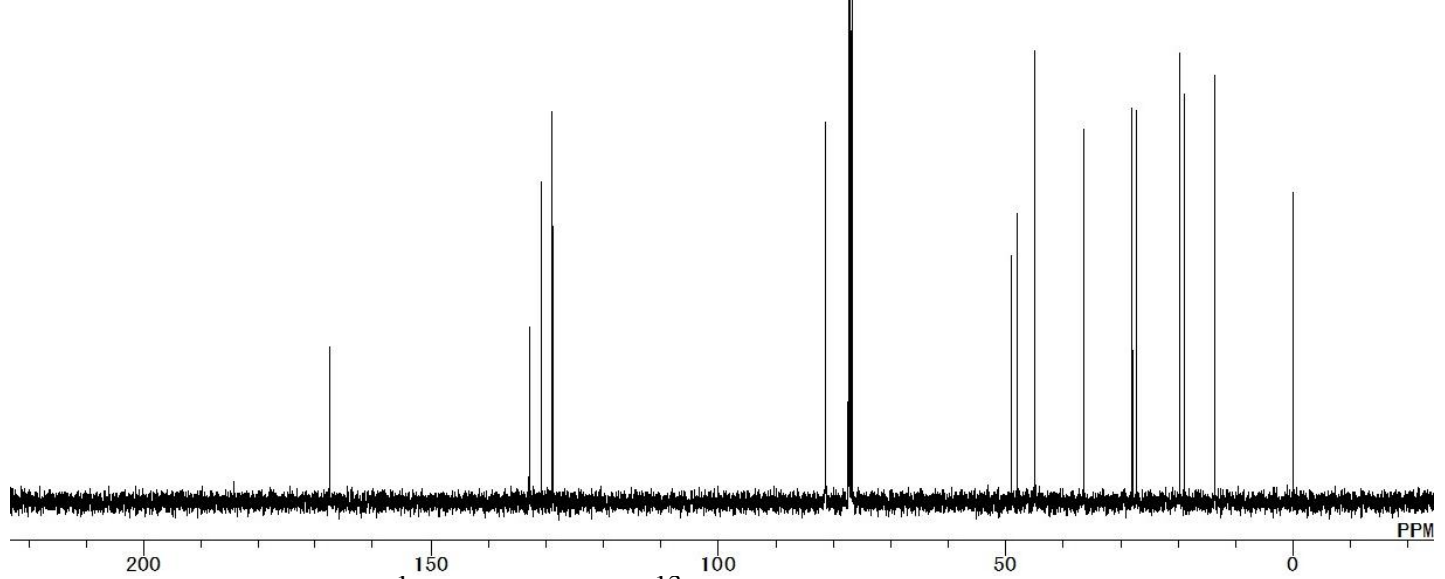

Fig. S4. ${ }^{1} \mathrm{H}$ (upper) and ${ }^{13} \mathrm{C}$ (lower) NMR spectra of I-o. 
Asymmetric Electrochemical Polymerization in Cholesteric Liquid Crystalline Media: Effect of Isomeric Structures of Chiral Inducers Containing Bornyl Group

Atsushi Matsumura, Fan Yang, Hiromasa Goto*

Synthetic Metals, 202, 157-164 (2015).

${ }^{1} \mathrm{H} \mathrm{NMR}\left(\mathrm{CDCl}_{3}, 400 \mathrm{MHz}\right)$

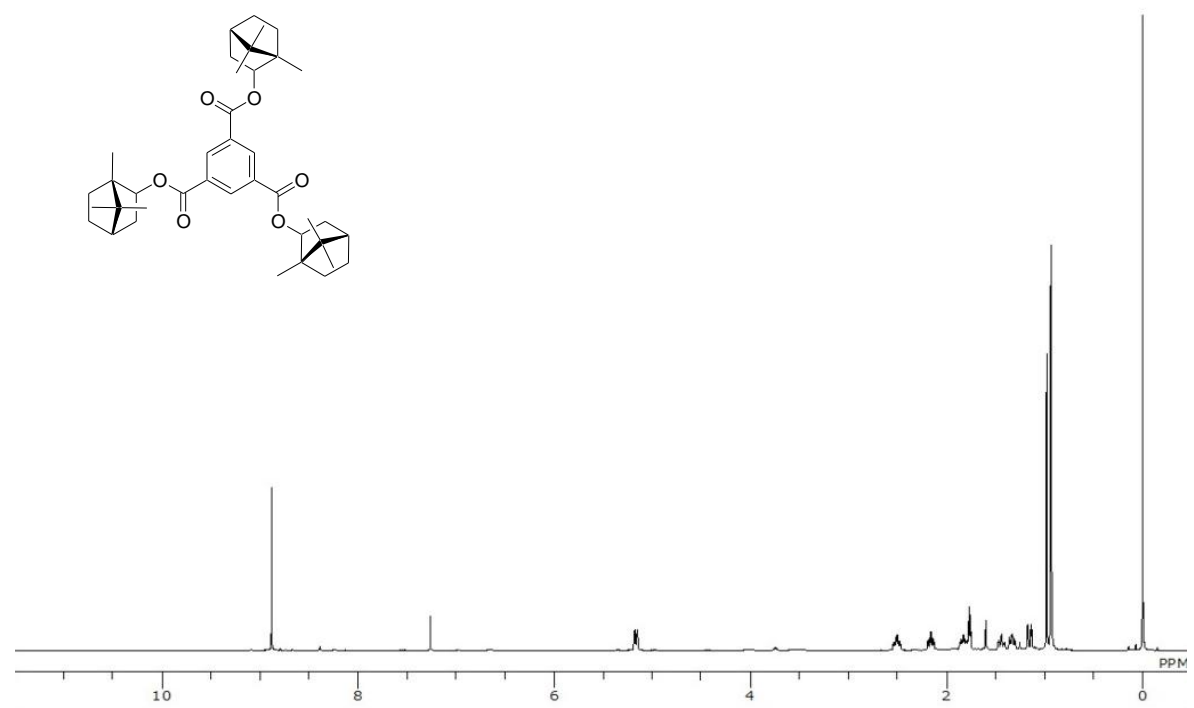

${ }^{13} \mathrm{C} \mathrm{NMR}\left(\mathrm{CDCl}_{3}, 100 \mathrm{MHz}\right)$

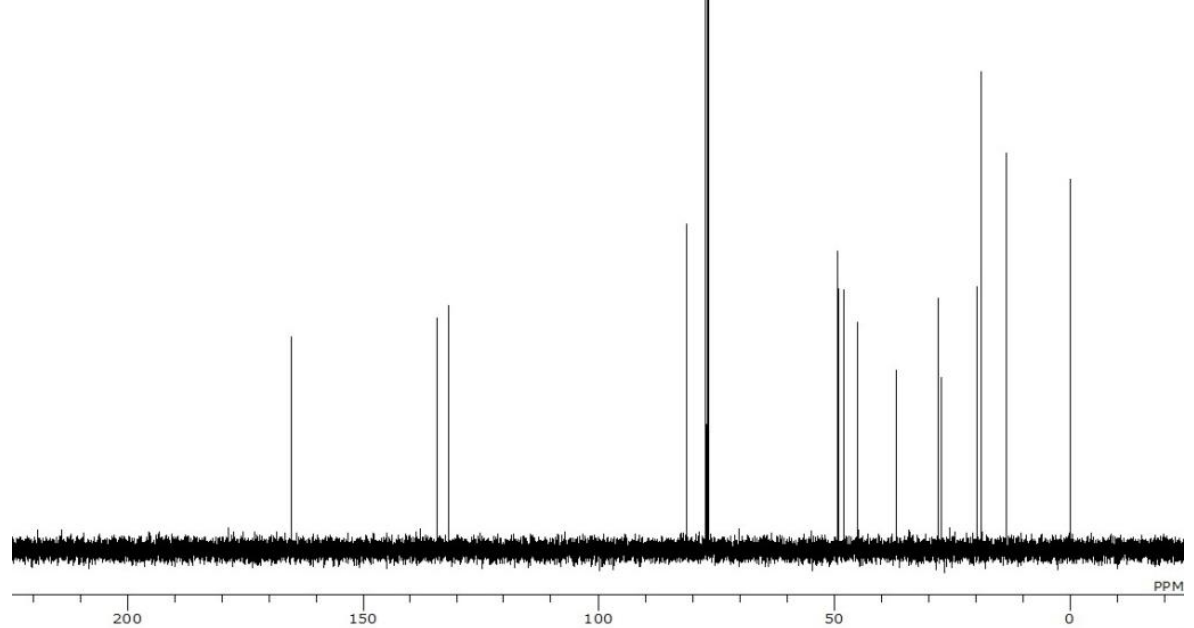

Fig. S5. ${ }^{1} \mathrm{H}$ (upper) and ${ }^{13} \mathrm{C}$ (lower) NMR spectra of I-t. 
Asymmetric Electrochemical Polymerization in Cholesteric Liquid Crystalline Media: Effect of Isomeric Structures of Chiral Inducers Containing Bornyl Group Atsushi Matsumura, Fan Yang, Hiromasa Goto*

Synthetic Metals, 202, 157-164 (2015).

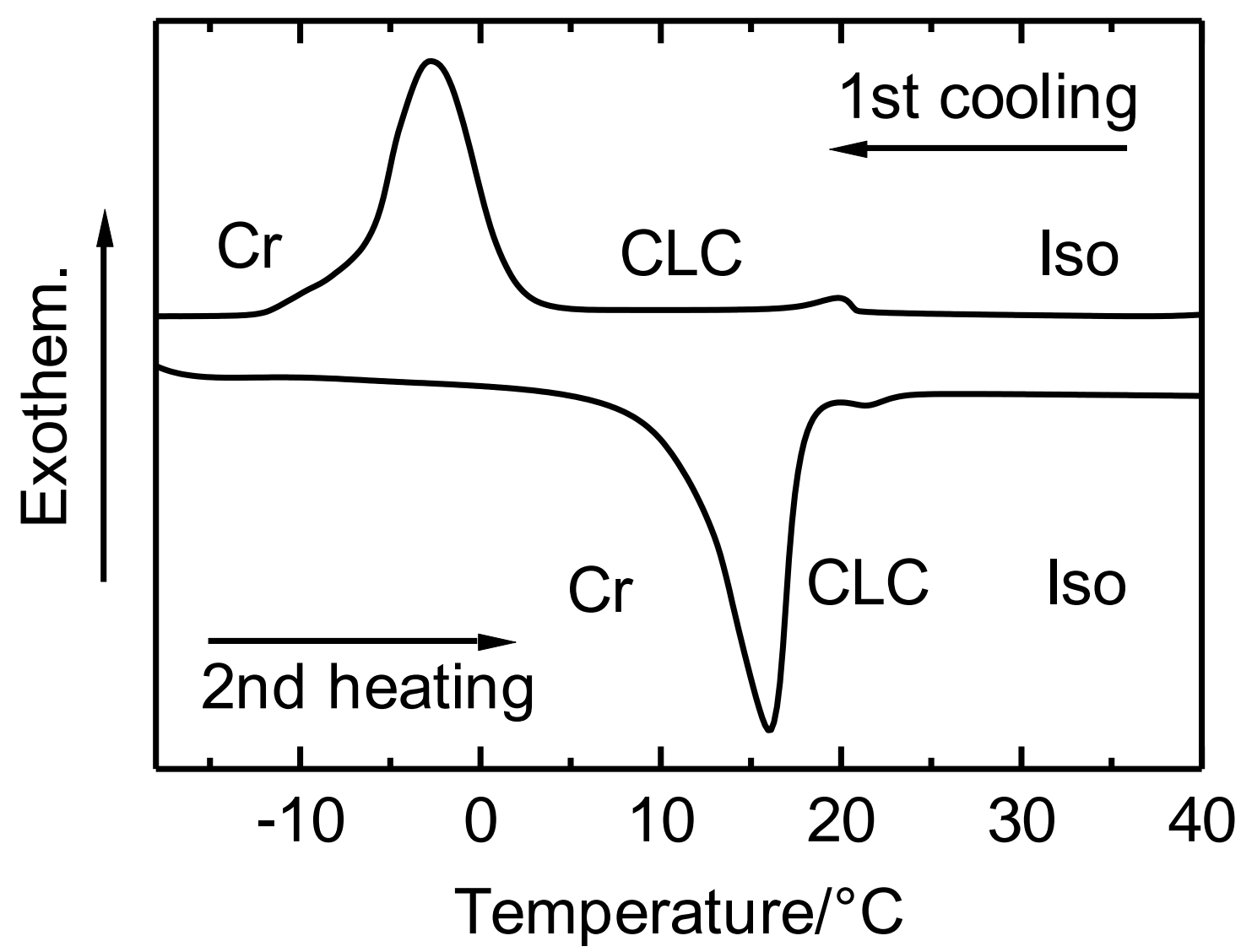

Fig. S6. Differential scanning calorimetry (DSC) curve for the CLC electrolyte solution induced by I-p. $\mathrm{Cr}$ and Iso denote crystal and isotropic phases, respectively. (DSC measurement was carried out with a rate of $10{ }^{\circ} \mathrm{C} \mathrm{min}^{-1}$ with EXSTRA7000 (Hitachi).) 
Asymmetric Electrochemical Polymerization in Cholesteric Liquid Crystalline Media: Effect of Isomeric Structures of Chiral Inducers Containing Bornyl Group

Atsushi Matsumura, Fan Yang, Hiromasa Goto*

Synthetic Metals, 202, 157-164 (2015).

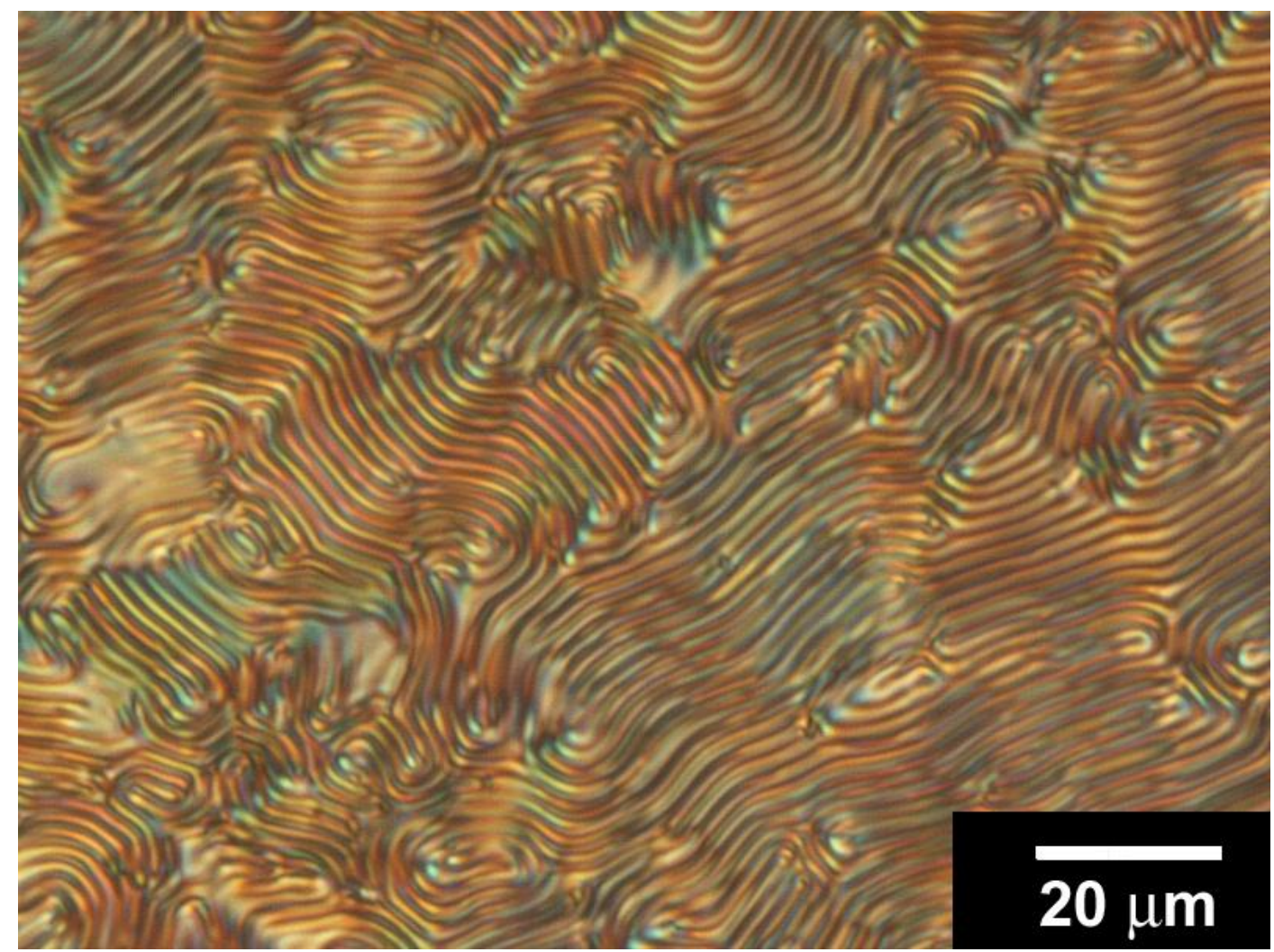

Fig. S7. Polarizing optical microscopy (POM) image of CLC electrolyte solution induced by I-p at $18^{\circ} \mathrm{C}$. 
Asymmetric Electrochemical Polymerization in Cholesteric Liquid Crystalline Media: Effect of Isomeric Structures of Chiral Inducers Containing Bornyl Group

Atsushi Matsumura, Fan Yang, Hiromasa Goto*

Synthetic Metals, 202, 157-164 (2015).

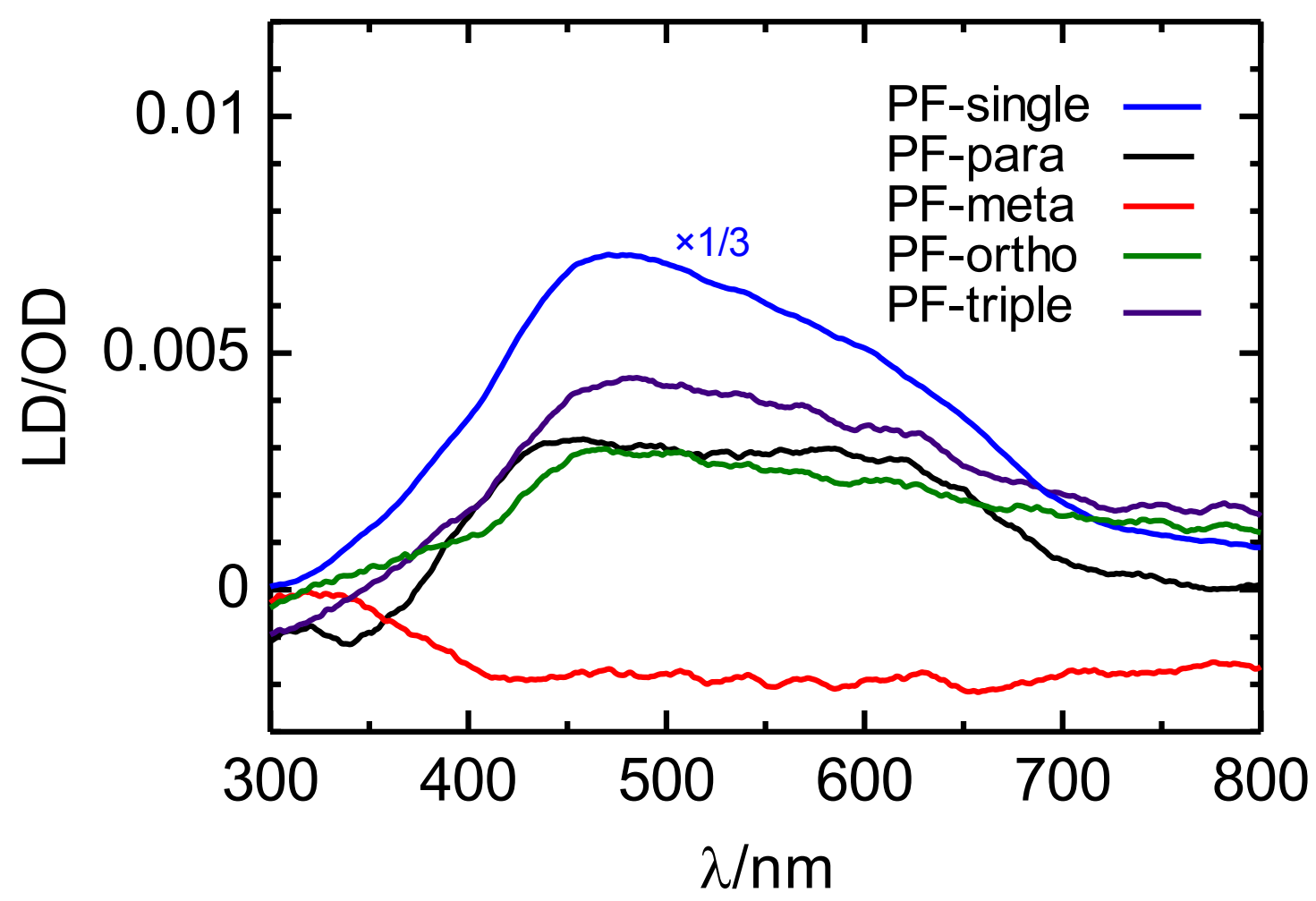

Fig. S8. LD spectra of the polymer films. 
Asymmetric Electrochemical Polymerization in Cholesteric Liquid Crystalline Media: Effect of Isomeric Structures of Chiral Inducers Containing Bornyl Group

Atsushi Matsumura, Fan Yang, Hiromasa Goto*

Synthetic Metals, 202, 157-164 (2015).

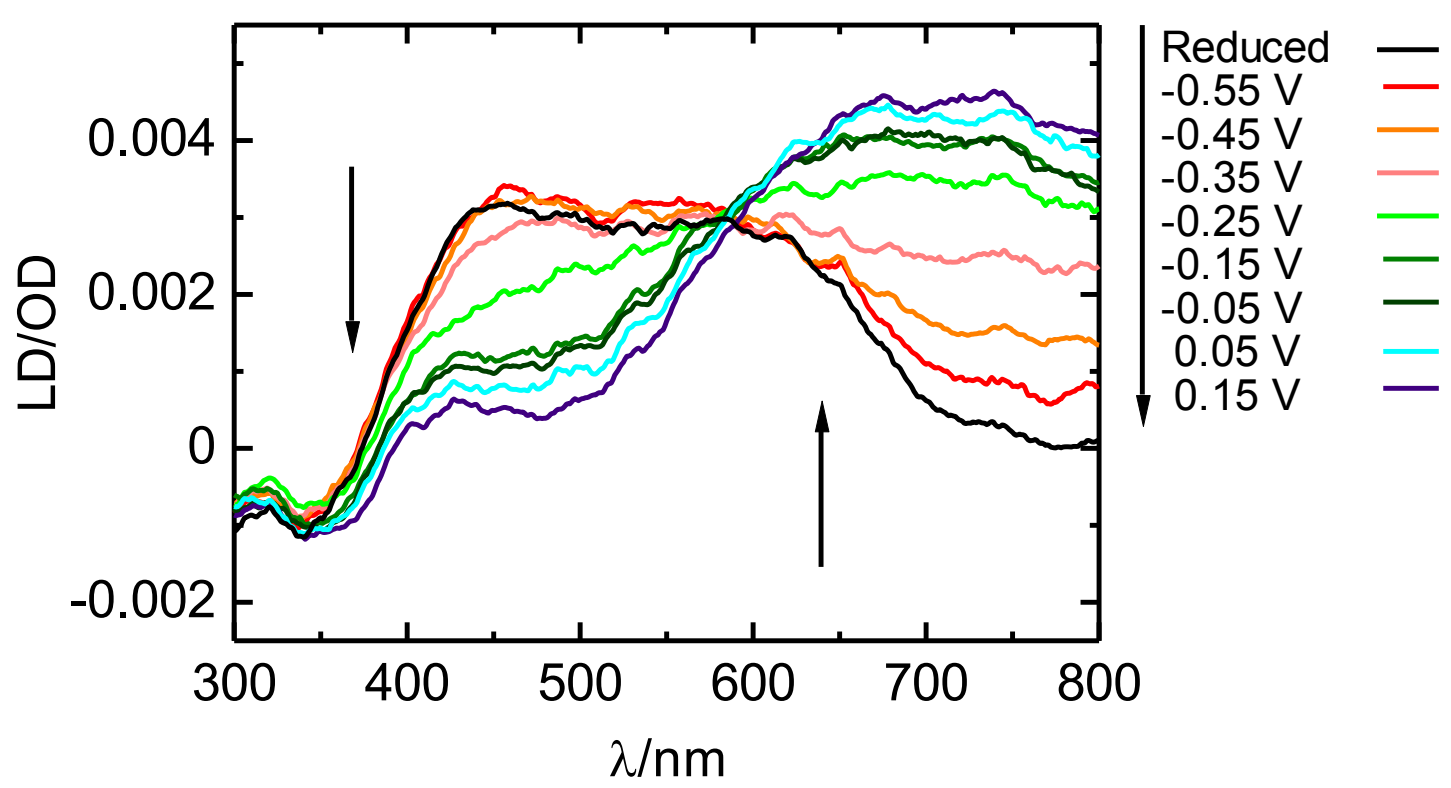

Fig. S9. LD spectra of PF-para during oxidation process. 